\title{
Non-holomorphic multi-matrix gauge invariant operators based on Brauer algebra
}

\author{
Yusuke Kimura \\ y.kimura@qmul.ac.uk \\ Centre for Research in String Theory, Department of Physics, \\ Queen Mary University of London, \\ Mile End Road, London E1 4NS UK
}

\begin{abstract}
We present an orthogonal basis of gauge invariant operators constructed from some complex matrices for the free matrix field, where operators are expressed with the help of Brauer algebra. This is a generalisation of our previous work for a signle complex matrix. We also discuss the matrix quantum mechanics relevant to $\mathcal{N}=4 \mathrm{SYM}$ on $S^{3} \times R$. A commuting set of conserved operators whose eigenstates are given by the orthogonal basis is shown by using enhanced symmetries at zero coupling.
\end{abstract}




\section{Introduction}

According to the AdS/CFT correspondence, gauge invariant operators correspond to physical states in string theory and, moreover, the spectrum of states is encoded in the anomalous dimension of gauge invariant operators. Of primary interest is to establish this correspondence exactly and to know how the space-time physics can be read from correlators of gauge invariant operators.

The half-BPS states have attracted much attention because of some observations. The chiral primary sector is described by the complex matrix model which can be obtained by the dimensional reduction from the original $\mathcal{N}=4 \mathrm{SYM}$, which leads to the free fermion description of this system [4, 5, 6, 7, 8, 9]. The chiral primary operators describe KK gravitons and giant gravitons [4, 10, 11, 12], whose two-point functions are diagonal [5]. One finds that a particular linear combination of single traces and multi-traces corresponds a single giant graviton or a set of giant gravitons, whose information is offered by Young tableau [5]. A Young diagram plays the role of organising the multi-trace structure of this sector and completely determines the space-time physics.

A next question along this line is how we can expand the chiral primary sector to more general classes of gauge invariant operators. It is interesting to ask how group theory can be utilised to organise gauge invariant operators to make the connection to string theory more manifest. In [1], the chiral primary operator was extended to include the complex conjugation of a complex matrix, and an orthogonal complete set was constructed using the free field correlator. Because replacing $X$ by $X^{\dagger}$ in chiral primary operators means to change the sign of angular momentum and the coupling to the background field in the dual string side, this sector would describe physics containing giant gravitons and anti-giant gravitons. If the interactions are taken into account properly, one may expect to see the instability originated from tachyons appearing between those branes.

A mathematical new element in [1] was to use Brauer algebra to organise the multi-trace structure of gauge invariant operators constructed from $X$ and $X^{\dagger}$. This is a generalisation of the fact that the symmetric group played a role to organise the multi-trace structure of the chiral primary sector. The Brauer algebra contains the contraction in addition to the group algebra of the symmetric group, which manages gauge invariant operators involving $X$ and $X^{\dagger}$ in a trace. The complete set constructed in [1] has more group theoretic labels than the chiral primary sector, which is reflected in the fact that the operators were conjectured to describe a system of branes and anti-branes. Other studies of diagonalising the two-point functions in the free field limit have been reported in [13, 14, 15, 16, 17].

In this paper, we continue this line to explore non-holomorphic gauge invariant operators built from some complex matrices. We work out the construction of diagonal two-point functions at the free level of the matrix field theory and see what kind of group theoretic structure show up.

Here are the outline of this paper. The construction of the orthogonal set of gauge invariant operators is reviewed in section 2 for the chiral primary sector and the nonholomorphic extension. In section 3 , we shall give the construction of an orthogonal set for 
the non-holomorphic multi-matrix sector. Section 4 is given to show some examples of our operator. The simplest sector contains the Konishi operator. In section 5 , the multi-matrix quantum mechanics is discussed. In our previous paper [3], we have introduced the concept of enhanced symmetries at the free level and have given some operators commuting with the Hamiltonian, which were exploited to measure the spectrum of orthogonal bases. It will be shown how the orthogonal gauge invariant operators constructed in this paper are characterised by conserved charges based on the enhanced symmetries. In section 6, we discuss some future problems. Some detailed calculations are shown in appendices.

\section{Diagonal basis of gauge invariant operators built from $X$ and $X^{\dagger}$ based on Brauer algebra : Review}

In this section, we review the chiral primary sector and the Brauer basis for $X$ and $X^{\dagger}$ sector.

Operators corresponding to the highest weight in the half-BPS sector are given by holomorphic gauge invariant operators made from a complex matrix $X$. The diagonal basis of operators constructed from $n$ matrices is labelled by a Young diagram $R$ with $n$ boxes [5]. We shall review the construction of this basis from a group theoretical point of view.

An $N \times N$ matrix $X$ can be viewed as an endomorphism acting on an $N$-dimensional vector space $V$, i.e. $X: V \rightarrow V$. The tensor product $X^{\otimes n}$ acts on $V^{\otimes n}$. It is useful to introduce the symmetric group $S_{n}$ as a tool to organise both single trace and multi-trace. We let elements $\sigma$ of the symmetric group $S_{n}$ act on $V^{\otimes n}$ as the permutations of $n$ vector spaces $V$. One easily finds that any gauge invariant operator constructed from $n X$ 's can be expressed as $\operatorname{tr}_{n}\left(\sigma X^{\otimes n}\right)=X_{i_{\sigma(1)}}^{i_{1}} \cdots X_{i_{\sigma(n)}}^{i_{n}}$ where the trace $t r_{n}$ is taken in $V^{\otimes n}$. It is noted that $\operatorname{tr}_{n}\left(h \sigma h^{-1} X^{\otimes n}\right)$ also gives the same gauge invariant operator for any $h \in S_{n}$, which means the conjugacy classes of $S_{n}$ classify gauge invariant operators.

It is convenient to start with the following Schur-Weyl duality to introduce the representation basis,

$$
V^{\otimes n}=\bigoplus_{R} V_{R}^{U(N)} \otimes V_{R}^{S_{n}}
$$

This comes from the fact that the symmetric group $S_{n}$ is the centraliser of $U(N)$ on $V^{\otimes n}$. $R$ runs over all irreducible representations with $n$ boxes satisfying $c_{1}(R) \leq N$, where $c_{1}(R)$ is the length of the first column of $R$. The projection operator associated with an irreducible representation $R$ can be expressed as an element in the group algebra of the symmetric group $S_{n}$ as

$$
p_{R}=\frac{d_{R}}{n !} \sum_{\sigma \in S_{n}} \chi_{R}(\sigma) \sigma .
$$

Consider the following particular linear combination of $\operatorname{tr}_{n}\left(\sigma X^{\otimes n}\right)$

$$
O_{R}(X):=\operatorname{tr}_{n}\left(p_{R} X^{\otimes n}\right) .
$$


It was shown in [5] that it has the diagonal two-point function 1:

$$
\left\langle O_{R}(X)^{\dagger} O_{S}(X)\right\rangle=\delta_{R S} n_{R} ! d_{R} \operatorname{Dim} R
$$

where $d_{R}$ and $\operatorname{Dim} R$ are the dimension of $S_{n}$ and that of $U(N)$ associated with the irreducible reprentation $R$. This two-point function can be shown using the propagator of $X_{i j}: 2$

$$
\left\langle X_{i j}^{\dagger} X_{k l}\right\rangle=\delta_{j k} \delta_{i l}
$$

The space-time dependence has been ignored because it is easily recovered from the conformal invariance, and we are mainly interested in the colour and flavour dependence. Some properties of the projector are very helpful to calculate correlation functions [18].

We extend this sector to include $X^{\dagger}$. The construction of an orthogonal set for nonholomorphic operators in the free matrix field theory was completed in [1] by introducing the Brauer algebra. The Brauer algebra is an algebra which has the group algebra of $S_{m} \times S_{n}$ and contractions 3. It is reviewed in section 3 of [1].

We start with the fact that any multi-trace gauge invariant operator constructed from $m$ copies of $X$ and $n$ copies of $X^{\dagger}$ can be indicated through an element of the Brauer algebra as $t r_{m, n}\left(b X^{\otimes m} \otimes X^{* \otimes n}\right)$, where $t r_{m, n}$ is taken in the space of $V^{\otimes m} \otimes \bar{V}^{\otimes n}$. At $m=n=1, b$ takes 1 (the unit element) and $C$ (contraction), which give $\operatorname{tr} X \operatorname{tr} X^{\dagger}$ and $\operatorname{tr}\left(X X^{\dagger}\right)$, respectively. We note that $t r_{m, n}\left(h^{-1} b h X^{\otimes m} \otimes X^{* \otimes n}\right)$ for any $h \in S_{m} \times S_{n}$ provides the same gauge invariant operator 4 . This means multi-trace operators are classified by the equivalence classes under the conjugation of the symmetric group $S_{m} \times S_{n}$.

The Brauer algebra 5 can be introduced as the centraliser of $U(N)$ acting on $V^{\otimes m} \otimes \bar{V}^{\otimes n}$,

$$
V^{\otimes m} \otimes \bar{V}^{\otimes n}=\bigoplus_{\gamma} V_{\gamma}^{U(N)} \otimes V_{\gamma}^{B_{N}(m, n)}
$$

The sum is over irreducible representations $\gamma$ of $U(N)$ and $B_{N}(m, n)$. This equation follows from the fact that Brauer elements commute with the action of $U(N)$ on $V^{\otimes m} \otimes \bar{V}^{\otimes n}$. The irreducible representation $\gamma$ is determined by a set $\left(\gamma_{+}, \gamma_{-}, k\right)$, where $\gamma_{+}$is a partition of $m-k, \gamma_{-}$is a partition of $n-k$ and $k$ is an integer with $0 \leq k \leq \min (m, n)$. These definitions provide a constraint $c_{1}\left(\gamma_{+}\right)+c_{1}\left(\gamma_{-}\right) \leq N$. Because the Wick contractions are symbolised in terms of the symmetric group $S_{m} \times S_{n}$ (see, e.g. (24)), it will be convenient

\footnotetext{
1 The two-point function was reproduced by $c=1$ tachyon scattering amplitudes in [19].

${ }^{2}$ In this paper, we study the theory with gauge group $U(N)$. See [20, 21] for the extension to gauge group $S U(N)$.

3 The contraction $C$ is a linear map from $V \otimes \bar{V}$ to itself. The action is $C v_{i} \otimes \bar{v}_{j}=\delta_{i j} v_{k} \otimes \bar{v}_{k}$.

${ }^{4}$ Using the cyclicity property of the trace, the conjugate action of $h$ on $b$ results in the conjugate action on $X^{\otimes m} \otimes X^{* \otimes n}$, which is re-ordering of $X^{\prime}$ 's and/or that of $X^{*}$ 's.

${ }^{5}$ The Brauer algebra is sensitive to $N$. For example, we have $C^{2}=N C$ for a contraction $C$.
} 
to decompose the Brauer algebra into the group algebra of $S_{m} \times S_{n}$ (which we shall denoted by $\left.\mathbb{C}\left(S_{m} \times S_{n}\right)\right)$ as

$$
V_{\gamma}^{B_{N}(m, n)}=\bigoplus_{A} V_{A}^{\mathbb{C}\left(S_{m} \times S_{n}\right)} \otimes V_{\gamma \rightarrow A}
$$

The sum is taken over irreducible representations $A$ of the symmetric group, and $V_{\gamma \rightarrow A}$ represents the space of the multiplicity associated with the decomposition. We shall express an irreducible representation $A$ of $S_{m} \times S_{n}$ as a set of a partition of $m$ and a partition of $n:(\alpha, \beta)$. The multiplicity of the irreducible representation $A=(\alpha, \beta)$ of $\mathbb{C}\left[S_{m} \times S_{n}\right]$ appearing in the irreducible representation $\gamma$ of $B_{N}(m, n)$ is read from the formula

$$
M_{A}^{\gamma}:=\operatorname{Dim}\left(V_{\gamma \rightarrow A}\right)=\sum_{\delta \vdash k} g\left(\delta, \gamma_{+} ; \alpha\right) g\left(\delta, \gamma_{-} ; \beta\right) .
$$

Here $\delta \vdash k$ is shorthand to express that $\delta$ is a partition of $k$. The Littlewood-Richardson coefficient $g\left(\delta, \gamma_{+} ; \alpha\right)$ represents the multiplicity of the representation $\alpha$ appearing in the tensor product of the representations $\delta$ and $\gamma_{+}$. This formula states $\gamma_{+}=\alpha$ and $\gamma_{-}=\beta$ at $k=0$.

An orthogonal and complete set introduced in [1] is

$$
O_{A, i j}^{\gamma}\left(X, X^{*}\right)=: \operatorname{tr}_{m, n}\left(Q_{A, i j}^{\gamma} X^{\otimes m} \otimes X^{* \otimes n}\right):
$$

where : means that divergences (self-contractions) associated with defining a composite operator are omitted, i.e. $\langle: \operatorname{tr}(\quad):\rangle=0$. The indices $i, j$ run over 1 to $M_{A}^{\gamma}$, behaving like matrix indices as $Q_{A, i j}^{\gamma} Q_{A^{\prime}, k l}^{\gamma^{\prime}}=\delta_{\gamma \gamma^{\prime}} \delta_{A A^{\prime}} \delta_{j k} Q_{A, i l}^{\gamma}$. When the multiplicity is trivial, this operator becomes a projector, which happens at $k=0, k=m=n$ [27], and so on.

We introduce the restricted character $\chi_{A, i j}^{\gamma}(b)$ which is defined by

$$
\chi_{A, i j}^{\gamma}(b)=\sum_{m_{A}}\left\langle\gamma \rightarrow A, m_{A}, i|b| \gamma \rightarrow A, m_{A}, j\right\rangle
$$

where $\left|\gamma \rightarrow A, m_{A}, j\right\rangle$ is a state of the Brauer algebra in the irreducible representation $\gamma$ associated with the decomposition to the subalgebra $\mathbb{C}\left(S_{m} \times S_{n}\right) . m_{A}$ represents components in $A . \chi_{\gamma}(b)=\sum_{A, i} \chi_{A, i i}^{\gamma}(b)$ is the character of the Brauer algebra. The restricted character enables us to express $Q_{A, i j}^{\gamma}$ in terms of elements in the Brauer algebra as 6

$$
Q_{A, i j}^{\gamma}=t_{\gamma} \sum_{b} \chi_{A, j i}^{\gamma}(b) b^{*}
$$

$t_{\gamma}$ is the dimension of the $U(N)$ irreducible representation $\gamma . b^{*}$ is the dual element of $b$ which is specified by $\operatorname{tr}_{m, n}\left(b b^{*}\right)=1$. For more information about this dual element, see [1, 43]. The operator $Q_{A, i j}^{\gamma}$ commutes with any element of $S_{m} \times S_{n}$ :

$$
h Q_{A, i j}^{\gamma}=Q_{A, i j}^{\gamma} h, \quad h \in \mathbb{C}\left(S_{m} \times S_{n}\right),
$$

\footnotetext{
${ }^{6}$ A typo in (122) of [3] is corrected here.
} 
which can be shown by exploiting $h b^{*} h^{-1}=\left(h b h^{-1}\right)^{*}$. The property (12) is significant to show the diagonal two-point function. A projector associated with an irreducible representation $\gamma$ can be written down as

$$
P^{\gamma}=t_{\gamma} \sum_{b} \chi_{\gamma}(b) b^{*}=\sum_{A, i} Q_{A, i i}^{\gamma}
$$

Some remarks are in order. The free two-point functions are shown to be diagonal:

$$
\left\langle O_{A, i j}^{\gamma}\left(X, X^{*}\right)^{\dagger} O_{A^{\prime}, i^{\prime} j^{\prime}}^{\gamma^{\prime}}\left(X, X^{*}\right)\right\rangle=m ! n ! d_{A} t_{\gamma} \delta_{\gamma \gamma^{\prime}} \delta_{A A^{\prime}} \delta_{i i^{\prime}} \delta_{j j^{\prime}},
$$

where $d_{A}$ is the dimension of $S_{m} \times S_{n}$ associated with the irreducible representation $A$.

There is a special sub-class of the operator $Q_{A, i j}^{\gamma}$, which is parametrised by $k=0$. In this case, $\gamma=A$, i.e. $\gamma_{+}=\alpha, \gamma_{-}=\beta$ (see the comment below (8) ). It is therefore labelled by two Young diagrams. We shall denote it by $P_{\alpha \beta}$. Because the leading term of $P_{\alpha \beta}$ is found to be $p_{\alpha} p_{\beta}$, this gives the product of the holomorphic operator and the anti-holomorphic operator $O_{\alpha}(X) O_{\beta}\left(X^{\dagger}\right)$. The $k=0$ projector $P_{\alpha \beta}$ also plays an important role in the context of the large $N$ expansion of two-dimensional Yang-Mills [22]. Using some properties of the Brauer algebra, a new expression of the $S U(N)$ dimension was given, leading to a new formulation of two-dimensional Yang-Mills [2].

This class of gauge invariant operators does not require the normal ordering prescription to make it well-defined as composite operators. In other words we can show

$$
: \operatorname{tr}_{m, n}\left(P_{\alpha \beta} X^{\otimes m} \otimes X^{* \otimes n}\right):=\operatorname{tr}_{m, n}\left(P_{\alpha \beta} X^{\otimes m} \otimes X^{* \otimes n}\right) .
$$

Considering the fact that the Wick contraction between an $X$ and an $X^{*}$ is performed by a contraction $C, C P_{\alpha \beta}=0$ is a sufficient condition for the above equation. It indeed follows from the fact that the projector $P_{\alpha \beta}$ is orthogonal to the other projectors relevant to $k \neq 0$.

We conclude this section by showing the simplest example of $m=n=1$ :

$$
\operatorname{tr} X \operatorname{tr} X^{\dagger}-\frac{1}{N} \operatorname{tr}\left(X X^{\dagger}\right), \quad \frac{1}{N} \operatorname{tr}\left(X X^{\dagger}\right)
$$

The first one is labelled by $k=0$ and $A=([1],[1])$, while the second one is by $\gamma=(\emptyset, \emptyset, 1)$ and $A=([1],[1])$.

\section{Diagonal basis of non-holomorphic multi-matrix gauge invariant operators}

In the previous section, we have reviewed a specific set of gauge invariant operators made from $X$ and $X^{\dagger}$ that are engineered by the Brauer algebra. In this section we generalise this to multi-matrix models. We shall present an orthogonal set of operators composed of $X_{a}$ and $X_{a}^{\dagger}$, where $a=1, \cdots, p$, at the free coupling. This has an additional flavour index 
compared to the previous case. Hence we first work out the flavour structure, and later we will move to the colour structure. This procedure exploits the approach of [13] to deal with global indices. When $p$ is 3 , it is relevant to the $S O(6)$ sector of $\mathcal{N}=4$ four-dimensional super Yang-Mills theory.

\subsection{Gauge covariant operators in representation basis}

We start with the following gauge covariant operator

$$
\left(O_{\vec{a}, \vec{b}}\right)_{J}^{I}:=\left(X_{a_{1}}\right)_{j_{1}}^{i_{1}} \otimes \cdots \otimes\left(X_{a_{m}}\right)_{j_{m}}^{i_{m}} \otimes\left(X_{b_{1}}^{*}\right)_{j_{m+1}}^{i_{m+1}} \otimes \cdots \otimes\left(X_{b_{n}}^{*}\right)_{j_{m+n}}^{i_{m+n}}
$$

where $a_{i}, b_{i}=1,2, \ldots, p$. We will rewrite the flavour structure using a representation basis.

Let $V_{F}$ be the space of the fundamental representation of $U(p)$. The following SchurWeyl duality is relevant to the flavour structure:

$$
V_{F}^{\otimes m} \otimes \bar{V}_{F}^{\otimes n}=\bigoplus_{\Lambda} V_{\Lambda}^{U(p)} \otimes V_{\Lambda}^{B_{p}(m, n)} .
$$

Here the sum runs over irreducible representations of $U(p)$ and $B_{p}(m, n)$. The representation $\Lambda$ is labelled by a set $\left(\Lambda_{+}, \Lambda_{-}, l\right)$, where $l$ is an integer with $0 \leq l \leq \min (m, n)$, and $\Lambda_{+}$ and $\Lambda_{-}$are given by a partition of $m-l$ and a partition of $n-l$. It is noted that this Brauer algebra $B_{p}(m, n)$ should not be confused with another Brauer algebra $B_{N}(m, n)$ which is relevant for the colour structure.

The group algebra of $S_{m} \times S_{n}, \mathbb{C}\left(S_{m} \times S_{n}\right)$, is a subalgebra of $B_{p}(m, n)$, hence we consider the decomposition of $B_{p}(m, n)$ into $\mathbb{C}\left(S_{m} \times S_{n}\right)$ :

$$
V_{\Lambda}^{B_{p}(m, n)}=\bigoplus_{\Lambda_{1}} V_{\Lambda_{1}}^{\mathbb{C}\left(S_{m} \times S_{n}\right)} \otimes V_{\Lambda \rightarrow \Lambda_{1}}
$$

where $\Lambda_{1}$ runs over irreducible representations of $\mathbb{C}\left(S_{m} \times S_{n}\right)$. The second factor in the right-hand side represents the space of the multiplicity arising from this decomposition. Combining (18) and (19), we have the following equation for the flavour structure

$$
V_{F}^{\otimes m} \otimes \bar{V}_{F}^{\otimes n}=\bigoplus_{\Lambda, \Lambda_{1}} V_{\Lambda}^{U(p)} \otimes V_{\Lambda_{1}}^{\mathbb{C}\left(S_{m} \times S_{n}\right)} \otimes V_{\Lambda \rightarrow \Lambda_{1}} .
$$

Based on this decomposition, one may introduce a covariant operator in a representation basis as

$$
O_{\vec{a}, \vec{b}}=\sum_{\Lambda, M_{\Lambda}, \Lambda_{1}, m_{\Lambda_{1}}, \tau} C_{\vec{a}, \vec{b}}^{\Lambda, M_{\Lambda}, \Lambda_{1}, m_{\Lambda_{1}}, \tau} O_{\Lambda, M_{\Lambda}, \Lambda_{1}, m_{\Lambda_{1}}, \tau}
$$

$M_{\Lambda}$ represents states in the irreducible representation $\Lambda$ of $U(p)$, and $m_{\Lambda_{1}}$ runs over states in the $\Lambda_{1} . \tau$ is an index running over the multiplicity of $\Lambda_{1}$ in $\Lambda$. The inverse is

$$
O_{\Lambda, M_{\Lambda}, \Lambda_{1}, m_{\Lambda_{1}, \tau}}=\sum_{\vec{a}, \vec{b}} C_{\Lambda, M_{\Lambda}, \Lambda_{1}, m_{\Lambda_{1}}, \tau}^{\vec{a}, \vec{b}} O_{\vec{a}, \vec{b}}
$$


We now calculate the free two-point function of the operator. Using

$$
\left\langle\left(X_{a}^{\dagger}\right)_{i j}\left(X_{b}\right)_{k l}\right\rangle=\delta_{a b} \delta_{j k} \delta_{i l}
$$

we get for $O_{\vec{a}, \vec{b}}$

$$
\left\langle:\left(O_{\vec{a}, \vec{b}}^{\dagger}\right)_{J}^{I}::\left(O_{\overrightarrow{a^{\prime}}, \overrightarrow{b^{\prime}}}\right)_{L}^{K}:\right\rangle=\sum_{\sigma \in S_{m} \times S_{n}} \prod_{k=1}^{m} \delta_{a_{k} a_{\sigma(k)}^{\prime}} \prod_{l=1}^{n} \delta_{b_{l} b_{\sigma(l)}^{\prime}}(\sigma)_{J}^{K}\left(\sigma^{-1}\right)_{L}^{I},
$$

where

$$
(\sigma)_{J}^{K}=(\sigma)_{j_{1} \cdots j_{m+n}}^{k_{1} \cdots k_{m+n}}:=\delta_{j_{\sigma(1)}}^{k_{1}} \cdots \delta_{j_{\sigma(m+n)}}^{k_{m+n}}
$$

The two-point function of the representation basis (22) can be computed as

$$
\begin{aligned}
& \left\langle:\left(O_{\Lambda, M_{\Lambda}, \Lambda_{1}, m_{\Lambda_{1}}, \tau}^{\dagger}\right)_{J}^{I}::\left(O_{\Lambda^{\prime}, M_{\Lambda^{\prime}}^{\prime}, \Lambda_{1}^{\prime}, m_{\Lambda_{1}^{\prime}}^{\prime}, \tau^{\prime}}\right)_{L}^{K}:\right\rangle \\
= & \delta_{\Lambda \Lambda^{\prime}} \delta_{M_{\Lambda} M_{\Lambda^{\prime}}^{\prime}} \delta_{\Lambda_{1} \Lambda_{1}^{\prime}} \delta_{\tau \tau^{\prime}} \sum_{\sigma \in S_{m} \times S_{n}} D_{m_{\Lambda_{1}} m_{\Lambda_{1}^{\prime}}^{\prime}}^{\Lambda_{1}}(\sigma)(\sigma)_{J}^{K}\left(\sigma^{-1}\right)_{L}^{I} .
\end{aligned}
$$

This will be proved in appendix B.1.

\subsection{Gauge invariant operators in representation basis}

All gauge invariant operators constructed from the covariant operators are expressed by $t r_{m, n}\left(b O_{\Lambda, M_{\Lambda}, \Lambda_{1}, m_{\Lambda_{1}}, \tau}\right)$. To rewrite the colour structure in terms of a representation basis, we again use the decomposition given in (6) and (7):

$$
V^{\otimes m} \otimes \bar{V}^{\otimes n}=\bigoplus_{\gamma, A} V_{\gamma}^{U(N)} \otimes V_{A}^{\mathbb{C}\left(S_{m} \times S_{n}\right)} \otimes V_{\gamma \rightarrow A}
$$

We thus have two kinds of irreducible representations of the symmetric group. One, $\Lambda$, is responsible for the global indices, and the other, $A$, is for the colour indices. These two kinds of representations come from two different actions of the symmetric group which are related each other as we shall discuss later (see (34) and appendix C). We now consider the inner tensor product of $A \otimes A$ decomposing into $\Lambda_{1}$ and denote the multiplicity of $\Lambda_{1}$ by $\tau_{\Lambda_{1}}$. Introducing the Clebsch-Gordan coefficient $7 C_{A, m_{A}, A, m_{A}^{\prime}}^{\tau_{\Lambda_{1}, \Lambda_{1}, m_{\Lambda_{1}}}}$ associated with this decomposition, we propose the following gauge invariant operator

$$
\mathcal{O}_{\Lambda, M_{\Lambda}, \Lambda_{1}, \tau}^{\gamma, A, i j \tau_{\Lambda_{1}}}=\sum_{m_{A}, m_{A}^{\prime}, m_{\Lambda_{1}}} C_{A, m_{A}, A, m_{A}^{\prime}}^{\tau_{\Lambda_{1}}, \Lambda_{1}, m_{\Lambda_{1}}} \sum_{b \in B_{N}(m, n)} D_{A, m_{A}, j, m_{A}^{\prime}, i}^{\gamma}\left(b^{*}\right): \operatorname{tr}_{m, n}\left(b O_{\Lambda, M_{\Lambda}, \Lambda_{1}, m_{\Lambda_{1}}, \tau}\right):
$$

Here

$$
D_{A, m_{A}, j, m_{A}^{\prime}, i}^{\gamma}(b):=\left\langle\gamma \rightarrow A, m_{A}, j|b| \gamma \rightarrow A, m_{A}^{\prime}, i\right\rangle
$$

\footnotetext{
7 See [23] for some properties of the Clebsch-Gordan coefficient.
} 
A remark is

$$
\chi_{A, j i}^{\gamma}(b)=\sum_{m_{A}} D_{A, m_{A}, j, m_{A}, i}^{\gamma}(b)
$$

is the restricted character in (10).

We can show that the two-point function of the operator (28) is diagonal:

$$
\left\langle\mathcal{O}_{\Lambda, M_{\Lambda}, \Lambda_{1}, \tau}^{\gamma, A, i j, \tau_{\Lambda_{1}} \dagger} \mathcal{O}_{\Lambda^{\prime}, M_{\Lambda^{\prime}}^{\prime}, \Lambda_{1}^{\prime}, \tau^{\prime}}^{\gamma^{\prime}, A^{\prime}, i^{\prime} j^{\prime}, \tau_{\Lambda^{\prime}}^{\prime}}\right\rangle=m ! n ! d_{\Lambda_{1}} \frac{1}{t^{\gamma}} \delta_{\gamma \gamma^{\prime}} \delta_{A A^{\prime}} \delta_{\Lambda \Lambda^{\prime}} \delta_{M_{\Lambda} M_{\Lambda^{\prime}}^{\prime}} \delta_{\Lambda_{1} \Lambda_{1}^{\prime}} \delta_{\tau_{\Lambda_{1}} \tau_{\Lambda_{1}^{\prime}}^{\prime}} \delta_{\tau \tau^{\prime}} \delta_{i i^{\prime}} \delta_{j j^{\prime}}
$$

This is the main result of this paper. We shall give the proof to the appendix B.

For $p=1$, the operator should coincide to the operator seen in the previous section. In this case, $\Lambda$ is a one-dimensional representation, so the covariant operator is labelled by $\Lambda$ alone i.e. the number of $X$ and that of $X^{*}$. The Clebsh-Gordan for this case is given by

$$
C_{A, m_{A}, A, m_{A}^{\prime}}=\frac{1}{\sqrt{d_{A}}} \delta_{m_{A} m_{A}^{\prime}} .
$$

We thus obtain

$$
\mathcal{O}_{\gamma, A, i j}=\frac{1}{\sqrt{d_{A}}} \sum_{b} \chi_{A, j i}^{\gamma}\left(b^{*}\right) \operatorname{tr}_{m, n}\left(b X^{\otimes m} \otimes X^{* \otimes n}\right),
$$

which is equivalent to (9) up to the normalisation factor.

Before proceeding to the next subsection, we summarise the relation between the two actions of the symmetric group. Two kinds of Brauer algebras have been introduced, one is relevant for the flavour structure, and the other is for the colour structure. Both Brauer algebras contain the same sub-algebra, that is, the group algebra of $S_{m} \times S_{n}$, but they admit different actions on the operator. The permutation acting on the flavour indices is equivalent to re-ordering of $X$ 's and/or that of $X^{*}$ 's. If we act with permutations on upper

colour indices and lower colour indices simultaneously, we effectively get permutations on flavour indices:

$$
\left(O_{\vec{a}, \vec{b}}\right)_{\sigma(J)}^{\sigma(I)}=\left(O_{\sigma(\vec{a}), \sigma(\vec{b})}\right)_{J}^{I}
$$

In appendix $\mathrm{C}$ we shall confirm that the gauge invariant operator respects this symmetry.

\subsection{Operator in the $k=0$ representation of $\gamma$}

Let us now study the special class of the operator where the representation of $\gamma$ is specified by $k=0$. The multiplicity indices related to the decomposition $\gamma \rightarrow A$ can go away because $\gamma=A$ from the formula (8) at $k=0$. So the operator is simplified to be

$$
\mathcal{O}_{\Lambda, M_{\Lambda}, \Lambda_{1}, \tau}^{A, \tau_{\Lambda_{1}}}=C_{A, m_{A}, A, m_{A}^{\prime}}^{\tau_{\Lambda_{1}}, \Lambda_{1}, m_{\Lambda_{1}}} \sum_{b \in B_{N}(m, n)} D_{m_{A}, m_{A}^{\prime}}^{A}(b) \operatorname{tr}_{m, n}\left(b^{*} O_{\Lambda, M_{\Lambda}, \Lambda_{1}, m_{\Lambda_{1}}, \tau}\right)
$$




$$
\begin{aligned}
& =C_{A, m_{A}, A, m_{A}^{\prime}}^{\tau_{\Lambda_{1}, \Lambda_{1}, m_{\Lambda_{1}}}} \sum_{\alpha \in S_{m} \times S_{n}} D_{m_{A}, m_{A}^{\prime}}^{A}(\alpha) t r_{m, n}\left(\alpha^{*} O_{\Lambda, M_{\Lambda}, \Lambda_{1}, m_{\Lambda_{1}}, \tau}\right) \\
& =C_{A, m_{A}, A, m_{A}^{\prime}}^{\tau_{\Lambda_{1}, \Lambda_{1}, m_{\Lambda_{1}}}} \sum_{\alpha \in S_{m} \times S_{n}}^{A} D_{m_{A}, m_{A}^{\prime}}^{A}(\alpha) t r_{m, n}\left(1^{*} \alpha^{-1} O_{\Lambda, M_{\Lambda}, \Lambda_{1}, m_{\Lambda_{1}}, \tau}\right) .
\end{aligned}
$$

$D_{m_{A}, m_{A}^{\prime}}^{A}(C)=0$ has been used to get the second equality. The third equality follows from the formula $\alpha^{*}=\left(1^{*}\right) \alpha^{-1}$ for $\alpha \in S_{m} \times S_{n}$ which is derived in [1].

For the complete set of the $X$ and $X^{\dagger}$ sector, we could show that operators corresponding to the $k=0$ representation do not require the normal ordering prescription because short distance singularities arising from self-contractions vanish. This property also holds in this case. Using the fact that the Wick contraction between an $X$ and an $X^{*}$ can be expressed by a contraction $C$, we show that replacing a set of an $X$ and an $X^{*}$ by a contraction $C$ in (35) vanishes:

$$
\begin{aligned}
& \sum_{\alpha} D_{m_{A}, m_{A}^{\prime}}^{A}(\alpha) t r_{m, n}\left(C 1^{*} \alpha^{-1} O^{\prime}\right) \\
= & \sum_{\alpha}^{\alpha} D_{m_{A}, m_{A}^{\prime}}^{A}(\alpha) \sum_{\gamma^{\prime}} \chi^{\gamma^{\prime}}\left(C 1^{*} \alpha^{-1}\right) t r_{m, n}\left(P^{\gamma^{\prime}} O^{\prime}\right) \\
= & \sum_{\alpha} \sum_{\gamma^{\prime}} D_{m_{A}, m_{A}^{\prime}}^{A}(\alpha) D_{I J}^{\gamma^{\prime}}\left(C 1^{*}\right) D_{J I}^{\gamma^{\prime}}\left(\alpha^{-1}\right) t r_{m, n}\left(P^{\gamma^{\prime}} O^{\prime}\right) \\
\propto & D_{m_{A}, m_{A}^{\prime}}^{A}\left(C 1^{*}\right)=0 .
\end{aligned}
$$

Here the first equality comes from the decomposition (66), and we have used the orthogonality of the representation matrix (B.14) in the last step. In this way, we can demonstrate that the normal ordering prescription is no longer needed for gauge invariant operators belonging to the $k=0$ representation.

Another property of the $k=0$ sector is that the leading term contains the product of a holomorphic operator and an anti-holomorphic operator. We shall show that the operator constructed in [13] will be reproduced as a subset of our operator. To show this, we use an explicit expression of $1^{*}$ :

$$
1^{*}=\frac{1}{N^{m+n}} \Omega_{m}^{-1} \Omega_{n}^{-1}+\cdots,
$$

where $\Omega_{m}$ is a central element in the group algebra of $S_{m}$, and $\cdots$ are terms which are not in $\mathbb{C}\left(S_{m} \times S_{n}\right)$ but in $B_{N}(m, n)$. See appendix $A$ in [1] about more concrete form of $1^{*}$ for some examples. We substitute the leading term of $1^{*}$ into (35) and restrict to the $l=0$ sector of the representation $\Lambda$. This implies we do not see terms in which flavour indices are contracted between $X$ and $X^{\dagger}$. This is the case of $\Lambda=\Lambda_{1}$, so the index $\tau$ can be suppressed.

The operator (35) with the above restrictions taken into account becomes

$$
\frac{1}{N^{m+n}} C_{A, m_{A}, A, m_{A}^{\prime}}^{\tau_{\Lambda_{1}}, \Lambda_{1}, m_{\Lambda_{1}}} \sum_{\alpha \in S_{m} \times S_{n}} D_{m_{A}, m_{A}^{\prime}}^{A}(\alpha) \operatorname{tr}_{m, n}\left(\Omega_{m}^{-1} \Omega_{n}^{-1} \alpha^{-1} O_{\Lambda_{1}, m_{\Lambda_{1}}}\right)
$$




$$
\begin{aligned}
& =\frac{1}{N^{m+n}} C_{A, m_{A}, A, m_{A}^{\prime}}^{\tau_{\Lambda_{1}}, \Lambda_{1}, m_{\Lambda_{1}}} \sum_{\alpha \in S_{m} \times S_{n}} D_{m_{A}, m_{A}^{\prime}}^{A}\left(\Omega_{m}^{-1} \Omega_{n}^{-1} \alpha\right) \operatorname{tr} r_{m, n}\left(\alpha^{-1} O_{\Lambda_{1}, m_{\Lambda_{1}}}\right) \\
& =\frac{1}{N^{m+n}} C_{A, m_{A}, A, m_{A}^{\prime}}^{\tau_{\Lambda_{1}}, \Lambda_{1}, m_{\Lambda_{1}}} \frac{1}{d_{A}} \chi_{A}\left(\Omega_{m}^{-1} \Omega_{n}^{-1}\right) \sum_{\alpha \in S_{m} \times S_{n}} D_{m_{A}, m_{A}^{\prime}}^{A}(\alpha) t r_{m, n}\left(\alpha^{-1} O_{\Lambda_{1}, m_{\Lambda_{1}}}\right) \\
& =\frac{1}{N^{m+n}} C_{A, m_{A}, A, m_{A}^{\prime}}^{\tau_{\Lambda_{1}, \Lambda_{1}, m_{\Lambda_{1}}}} \frac{1}{d_{A}} \chi_{A}\left(\Omega_{m}^{-1} \Omega_{n}^{-1}\right) \sum_{\sigma \in S_{m}, \tau \in \times S_{n}} D_{m_{A}, m_{A}^{\prime}}^{A}(\sigma \otimes \tau) \operatorname{tr} m, n \\
&
\end{aligned}
$$

Each factor can be factorised into the $S_{m}$ part and the $S_{n}$ part. For example, the character can be rewritten as

$$
\begin{aligned}
\chi_{A}\left(\Omega_{m}^{-1} \Omega_{n}^{-1}\right) & =\chi_{R}\left(\Omega_{m}^{-1}\right) \chi_{S}\left(\Omega_{n}^{-1}\right) \\
& =\frac{1}{m ! n !} \frac{N^{m+n} d_{R}^{2} d_{S}^{2}}{\operatorname{DimRDimS}},
\end{aligned}
$$

where we have expressed $A$ as $(R, S)$ where $R$ is an irreducible representation of $S_{m}$ and $S$ is an irreducible representation of $S_{n}$, and the formula $\operatorname{Dim} R=\frac{N^{m}}{m !} \chi_{R}\left(\Omega_{m}\right)$ has been used. Taking this factorisation into account, we find that the operator in the $k=0$ representation of $\gamma$ and the $l=0$ representation of $\Lambda$ contains the product of the holomorphic multi-matrix operator and the anti-holomorphic multi-matirx operator presented in [13, 17].

\section{Examples}

In this section, we present explicit forms of our operator for two simple cases. We will set $p=3$ to see a connection to the $\mathcal{N}=4 \mathrm{SYM}$.

\section{$4.1 m=1, n=1$}

In this case, there are only two cases for $\Lambda=\left(\Lambda_{+}, \Lambda_{-}, l\right)$, i.e. $([1],[1], 0)$ and $(\emptyset, \emptyset, 1)$. We call them $l=0$ and $l=1$ because the integer $l$ completely identifies them.

The bases $\left|\Lambda, M_{\Lambda}\right\rangle$ are

$$
\begin{aligned}
& |l=0\rangle=|a, b\rangle-\frac{1}{3} \delta_{a b} \sum_{c=1}^{3}|c, c\rangle, \\
& |l=1\rangle=\frac{1}{3} \delta_{a b} \sum_{c=1}^{3}|c, c\rangle .
\end{aligned}
$$

Using the above states, the Clebsch-Gordan coefficient $C_{\Lambda, M_{\Lambda}}^{a^{\prime}, b^{\prime}}=\left\langle a^{\prime}, b^{\prime} \mid \Lambda, M_{\Lambda}\right\rangle$ can be calculated as

$$
\begin{aligned}
& C_{l=0}^{a^{\prime}, b^{\prime}}=\left\langle a^{\prime}, b^{\prime} \mid l=0\right\rangle=\delta_{a a^{\prime}} \delta_{b b^{\prime}}-\frac{1}{3} \delta_{a b} \delta_{a^{\prime} b^{\prime}}, \\
& C_{l=1}^{a^{\prime}, b^{\prime}}=\left\langle a^{\prime}, b^{\prime} \mid l=1\right\rangle=\frac{1}{3} \delta_{a b} \delta_{a^{\prime} b^{\prime}},
\end{aligned}
$$


which yield covariant operators $O_{\Lambda, M_{\Lambda}}=C_{\Lambda, M_{\Lambda}}^{a^{\prime}, b^{\prime}}\left(X_{a^{\prime}} \otimes X_{b^{\prime}}\right)$ :

$$
\begin{aligned}
O_{l=0} & =X_{a} \otimes X_{b}^{*}-\frac{1}{3} \delta_{a b} \sum_{c^{\prime}} X_{c^{\prime}} \otimes X_{c^{\prime}}^{*}, \\
O_{l=1} & =\frac{1}{3} \delta_{a b} \sum_{c^{\prime}} X_{c^{\prime}} \otimes X_{c^{\prime}}^{*} .
\end{aligned}
$$

We next work out the gauge invariant operator. For this case the operator (28) is simplified to

$$
\mathcal{O}_{\Lambda, M_{\Lambda}}^{\gamma}=\sum_{b \in B_{N}(1,1)} D^{\gamma}\left(b^{*}\right) \operatorname{tr}_{1,1}\left(b O_{\Lambda, M_{\Lambda}}\right)=\frac{1}{t_{\gamma}} \operatorname{tr}_{1,1}\left(P^{\gamma} O_{\Lambda, M_{\Lambda}}\right) .
$$

$\gamma$ is specified by an integer $k$, which takes either $k=0$ or $k=1$. $P^{\gamma}$ is a projector associated with $\gamma$ :

$$
P^{(k=0)}=1-\frac{C}{N}, \quad P^{(k=1)}=\frac{C}{N} .
$$

Re-normalising the operators as $\mathcal{O}^{\gamma} \rightarrow t_{\gamma} \mathcal{O}^{\gamma}$ for convenience, we reach the following gauge invariant operators

$$
\begin{aligned}
& \mathcal{O}_{k=0, l=0}=\left(\operatorname{tr} X_{a}\right)\left(\operatorname{tr} X_{b}^{\dagger}\right)-\frac{1}{N} \operatorname{tr}\left(X_{a} X_{b}^{\dagger}\right)-\frac{1}{3} \delta_{a b}\left(\operatorname{tr} X_{c}\right)\left(\operatorname{tr} X_{c}^{\dagger}\right)+\frac{1}{3 N} \delta_{a b} \operatorname{tr}\left(X_{c} X_{c}^{\dagger}\right) \\
& \mathcal{O}_{k=1, l=0}=\frac{1}{N}\left(\operatorname{tr}\left(X_{a} X_{b}^{\dagger}\right)-\frac{1}{3} \delta_{a b} \operatorname{tr}\left(X_{c} X_{c}^{\dagger}\right)\right) \\
& \mathcal{O}_{k=0, l=1}=\frac{1}{3} \delta_{a b}\left(\left(\operatorname{tr} X_{c}\right)\left(\operatorname{tr} X_{c}^{\dagger}\right)-\frac{1}{N} \operatorname{tr}\left(X_{c} X_{c}^{\dagger}\right)\right) \\
& \mathcal{O}_{k=1, l=1}=\frac{1}{3 N} \delta_{a b} \operatorname{tr}\left(X_{c} X_{c}^{\dagger}\right) .
\end{aligned}
$$

In fact, these are not eigenstates of the one-loop dilatation operator 8 . Correct eigenstates at one-loop are

$$
\begin{aligned}
& \mathcal{O}_{k=0, l=0} \\
& \mathcal{O}_{k=1, l=0} \\
& \mathcal{O}_{k=0, l=1}+\mathcal{O}_{k=1, l=1}=\frac{1}{3} \delta_{a b}\left(\operatorname{tr} X_{c}\right)\left(\operatorname{tr} X_{c}^{\dagger}\right) \\
& \frac{N^{2}-1}{N} \mathcal{O}_{k=1, l=1}-\frac{1}{N} \mathcal{O}_{k=0, l=1}=\frac{1}{3} \delta_{a b}\left(\operatorname{tr}\left(X_{c} X_{c}^{\dagger}\right)-\frac{1}{N}\left(\operatorname{tr} X_{c}\right)\left(\operatorname{tr} X_{c}^{\dagger}\right)\right)
\end{aligned}
$$

with eigenvalues $0,0,0,6 / \sqrt{3}$, respectively. The first three are operators in the short multiplets. The last one is the Konishi operator. The $l=0$ representations are not mixed up with the $l=1$ representations because the Hamiltonian commutes with the Brauer algebra governing the flavour structure.

\footnotetext{
${ }^{8}$ The one-loop dilatation operator in the so(6) sector will be rewritten in terms of the complex variables in appendix $\mathrm{A}$.
} 


\section{$4.2 m=2, n=1$}

We next show the case at $m=2, n=1$. States $\left|\Lambda, \Lambda_{1}\right\rangle$ are

$$
\begin{aligned}
|[2],[1], 0\rangle= & \frac{1}{2}(|a, b, c\rangle+|b, a, c\rangle)-\frac{1}{8} \delta_{a c} \sum_{d=1}^{3}(|d, b, d\rangle+|b, d, d\rangle) \\
& -\frac{1}{8} \delta_{b c} \sum_{d=1}^{3}(|a, d, d\rangle+|d, a, d\rangle) \\
|[1,1],[1], 0\rangle= & \frac{1}{2}(|a, b, c\rangle-|b, a, c\rangle)-\frac{1}{4} \delta_{a c} \sum_{d=1}^{3}(|d, b, d\rangle-|b, d, d\rangle) \\
|([1], \emptyset, 1),([2],[1])\rangle= & \frac{1}{8} \delta_{a c} \sum_{d=1}^{3}(|d, b, d\rangle+|b, d, d\rangle)+\frac{1}{8} \delta_{b c} \sum_{d=1}^{3}(|a, d, d\rangle+|d, a, d\rangle) \\
|([1], \emptyset, 1),([1,1],[1])\rangle= & \frac{1}{4} \delta_{a c} \sum_{d=1}^{3}(|d, b, d\rangle-|b, d, d\rangle)+\frac{1}{4} \delta_{b c} \sum_{d=1}^{3}(|a, d, d\rangle-|d, a, d\rangle) .
\end{aligned}
$$

Projectors in (49) and (51) with $N$ replaced by $3(=p)$ are useful to calculate the above equations. Recall that $\Lambda=\Lambda_{1}$ for the $l=0$ representation. Then the Clebsch-Gordan coefficients are

$$
\begin{aligned}
C_{[2],[1], l=0}^{a^{\prime}, b^{\prime}}= & \frac{1}{2}\left(\delta_{a a^{\prime}} \delta_{b b^{\prime}} \delta_{c c^{\prime}}+\delta_{a b^{\prime}} \delta_{b a^{\prime}} \delta_{c c^{\prime}}\right)-\frac{1}{8} \delta_{a c}\left(\delta_{a^{\prime} c^{\prime}} \delta_{b b^{\prime}}+\delta_{b^{\prime} c^{\prime}} \delta_{b a^{\prime}}\right) \\
& -\frac{1}{8} \delta_{b c}\left(\delta_{b^{\prime} c^{\prime}} \delta_{a a^{\prime}}+\delta_{a^{\prime} c^{\prime}} \delta_{a b^{\prime}}\right) \\
C_{[1,1],[1], l=0}^{a^{\prime}, b^{\prime}}= & \frac{1}{2}\left(\delta_{a a^{\prime}} \delta_{b b^{\prime}} \delta_{c c^{\prime}}-\delta_{a b^{\prime}} \delta_{b a^{\prime}} \delta_{c c^{\prime}}\right)-\frac{1}{4} \delta_{a c}\left(\delta_{a^{\prime} c^{\prime}} \delta_{b b^{\prime}}-\delta_{b^{\prime} c^{\prime}} \delta_{b a^{\prime}}\right) \\
& -\frac{1}{4} \delta_{b c}\left(\delta_{b^{\prime} c^{\prime}} \delta_{a a^{\prime}}-\delta_{a^{\prime} c^{\prime}} \delta_{a b^{\prime}}\right) \\
C_{l=1, \Lambda_{1}=([2],[1])=}^{a^{\prime}, b^{\prime}}= & \frac{1}{8} \delta_{a c}\left(\delta_{b b^{\prime}} \delta_{a^{\prime} c^{\prime}}+\delta_{b a^{\prime}} \delta_{b^{\prime} c^{\prime}}\right)+\frac{1}{8} \delta_{b c}\left(\delta_{a a^{\prime}} \delta_{b^{\prime} c^{\prime}}+\delta_{a b^{\prime}} \delta_{a^{\prime} c^{\prime}}\right) \\
C_{l=1, \Lambda_{1}=([1,1],[1])=}^{a^{\prime}, b^{\prime}}= & \frac{1}{4} \delta_{a c}\left(\delta_{b b^{\prime}} \delta_{a^{\prime} c^{\prime}}-\delta_{b a^{\prime}} \delta_{b^{\prime} c^{\prime}}\right)+\frac{1}{4} \delta_{b c}\left(\delta_{a a^{\prime}} \delta_{b^{\prime} c^{\prime}}-\delta_{a b^{\prime}} \delta_{a^{\prime} c^{\prime}}\right),
\end{aligned}
$$

giving rise to the following covariant operators

$$
\begin{aligned}
& O_{[2][1], l=0}= \frac{1}{2}\left(X_{a} \otimes X_{b} \otimes X_{c}^{*}+X_{b} \otimes X_{a} \otimes X_{c}^{*}\right)-O_{l=1, \Lambda_{1}=([2][1])} \\
& O_{[1,1][1], l=0}= \frac{1}{2}\left(X_{a} \otimes X_{b} \otimes X_{c}^{*}-X_{b} \otimes X_{a} \otimes X_{c}^{*}\right)-O_{l=1, \Lambda_{1}=([1,1][1])} \\
& O_{l=1, \Lambda_{1}=([2][1])=}=\frac{1}{8} \delta_{a c}\left(X_{c^{\prime}} \otimes X_{b} \otimes X_{c^{\prime}}^{*}+X_{b} \otimes X_{c^{\prime}} \otimes X_{c^{\prime}}^{*}\right) \\
& \\
&+\frac{1}{8} \delta_{b c}\left(X_{a} \otimes X_{c^{\prime}} \otimes X_{c^{\prime}}^{*}+X_{c^{\prime}} \otimes X_{a} \otimes X_{c^{\prime}}^{*}\right)
\end{aligned}
$$




$$
\begin{aligned}
O_{l=1, \Lambda_{1}=([1,1][1])=} & \frac{1}{4} \delta_{a c}\left(X_{c^{\prime}} \otimes X_{b} \otimes X_{c^{\prime}}^{*}-X_{b} \otimes X_{c^{\prime}} \otimes X_{c^{\prime}}^{*}\right) \\
& +\frac{1}{4} \delta_{b c}\left(X_{a} \otimes X_{c^{\prime}} \otimes X_{c^{\prime}}^{*}-X_{c^{\prime}} \otimes X_{a} \otimes X_{c^{\prime}}^{*}\right) .
\end{aligned}
$$

We next present gauge invariant operators. For the $k=0$ representation of $\gamma$, some labels in (28) are suppressed to give

$$
\mathcal{O}_{\Lambda}^{\gamma(k=0)}=\sum_{b \in B_{N}(2,1)} D^{\gamma}\left(b^{*}\right) \operatorname{tr}_{2,1}\left(b O_{\Lambda}\right)=\frac{1}{t^{\gamma}} \operatorname{tr}_{2,1}\left(P^{\gamma} O_{\Lambda}\right)
$$

where $P^{\gamma}$ is the central projection operator in the Brauer algebra, which is given in [1] by

$$
\begin{aligned}
& P_{[2][\overline{1}]}=\left(1-\frac{1}{N+1} C\right) p_{[2]}, \\
& P_{\left[1^{2}\right][\overline{1}]}=\left(1-\frac{1}{N-1} C\right) p_{\left[1^{2}\right]} .
\end{aligned}
$$

Here $C:=C_{1 \overline{1}}+C_{2 \overline{1}}$ commutes with any element in $\mathbb{C}\left(S_{2}\right)$. For the $k=1$ representation, we have

$$
\mathcal{O}_{\Lambda, \Lambda_{1}}^{\gamma(k=1), A}=\sum_{b \in B_{N}(2,1)} D_{A}^{\gamma}\left(b^{*}\right) \operatorname{tr}_{2,1}\left(b O_{\Lambda, \Lambda_{1}}\right)=\frac{1}{t^{\gamma}} \operatorname{tr}_{2,1}\left(P_{A}^{\gamma} O_{\Lambda, \Lambda_{1}}\right),
$$

where

$$
\begin{aligned}
& P_{[2][\overline{1}]}^{\left(k=1, \gamma_{+}=[1], \gamma_{-}=\emptyset\right)}=\frac{1}{N+1} C p_{[2]}, \\
& P_{\left[1^{2}\right][\overline{1}]}^{\left(k=1, \gamma_{+}=[1], \gamma_{-}=\emptyset\right)}=\frac{1}{N-1} C p_{\left[1^{2}\right]} .
\end{aligned}
$$

We exhibit some of them explicitly

$$
\begin{aligned}
& \operatorname{tr}_{2,1}\left(P_{[2][\overline{1}]} O_{[2][1], l=0}\right) \\
= & \frac{1}{2}\left(\operatorname{tr} X_{a} \operatorname{tr} X_{b} \operatorname{tr} X_{c}^{\dagger}+\operatorname{tr}\left(X_{a} X_{b}\right) \operatorname{tr} X_{c}^{\dagger}\right) \\
& -\frac{1}{2} \frac{1}{N+1}\left(\operatorname{tr} X_{a} \operatorname{tr}\left(X_{b} X_{c}^{\dagger}\right)+\operatorname{tr} X_{b} \operatorname{tr}\left(X_{a} X_{c}^{\dagger}\right)+\operatorname{tr}\left(X_{a} X_{b} X_{c}^{\dagger}\right)+\operatorname{tr}\left(X_{b} X_{a} X_{c}^{\dagger}\right)\right) \\
& -\operatorname{tr}_{2,1}\left(P_{[2][\overline{1}]} O_{l=1, \Lambda_{1}=[2][1]}\right), \\
& \operatorname{tr}_{2,1}\left(P_{[2][\overline{1}]} O_{l=1, \Lambda_{1}=[2][1]}\right) \\
= & \frac{1}{8} \delta_{a c}\left(\operatorname{tr} X_{b} \operatorname{tr} X_{c^{\prime}} \operatorname{tr} X_{c^{\prime}}^{\dagger}+\operatorname{tr}\left(X_{b} X_{c^{\prime}}\right) \operatorname{tr} X_{c^{\prime}}^{\dagger}\right) \\
& +\frac{1}{8} \delta_{b c}\left(\operatorname{tr} X_{a} \operatorname{tr} X_{c^{\prime}} \operatorname{tr} X_{c^{\prime}}^{\dagger}+\operatorname{tr}\left(X_{a} X_{c^{\prime}}\right) \operatorname{tr} X_{c^{\prime}}^{\dagger}\right) \\
& -\frac{1}{8} \frac{1}{N+1} \delta_{a c}\left(\operatorname{tr} X_{b} \operatorname{tr}\left(X_{c^{\prime}} X_{c^{\prime}}^{\dagger}\right)+\operatorname{tr} X_{c^{\prime}} \operatorname{tr}\left(X_{b} X_{c^{\prime}}^{\dagger}\right)+\operatorname{tr}\left(X_{b} X_{c^{\prime}} X_{c^{\prime}}^{\dagger}\right)+\operatorname{tr}\left(X_{b} X_{c^{\prime}}^{\dagger} X_{c^{\prime}}\right)\right) \\
& -\frac{1}{8} \frac{1}{N+1} \delta_{b c}\left(\operatorname{tr} X_{a} \operatorname{tr}\left(X_{c^{\prime}} X_{c^{\prime}}^{\dagger}\right)+\operatorname{tr} X_{c^{\prime}} \operatorname{tr}\left(X_{a} X_{c^{\prime}}^{\dagger}\right)+\operatorname{tr}\left(X_{a} X_{c^{\prime}} X_{c^{\prime}}^{\dagger}\right)+\operatorname{tr}\left(X_{a} X_{c^{\prime}}^{\dagger} X_{c^{\prime}}\right)\right) .
\end{aligned}
$$




\section{Quantum mechanics and conserved charges measur- ing representation labels}

In this section, we study the matrix quantum mechanics which is obtained by the dimensional reduction of the four-dimensional $\mathcal{N}=4 \mathrm{SYM}$ on $S^{3} \times R$. Because we are mainly interested in the free theory, we ignore the interaction terms. It is found that the Hamiltonian can be given by a set of the harmonic oscillators [5], and it has been known that the harmonic oscillator is characterised by many conserved charges. Some conserved charges whose eigenvalues can specify an orthogonal state were constructed in [3]. Furthermore, the construction was based on symmetries which are enhanced at the free level. In this section, we show conserved charges for the present context.

The Hamiltonian and $U(1)^{3}$ charge are

$$
\begin{aligned}
& H=\sum_{a} \operatorname{tr}\left(A_{a}^{\dagger} A_{a}+B_{a}^{\dagger} B_{a}\right)+N^{2}, \\
& J_{a}=\operatorname{tr}\left(B_{a}^{\dagger} B_{a}-A_{a}^{\dagger} A_{a}\right),
\end{aligned}
$$

where we have introduced the matrix annihilation and creation operators with the non-zero commutation relations

$$
\left[\left(A_{a}\right)_{i j},\left(A_{b}^{\dagger}\right)_{k l}\right]=\delta_{a b} \delta_{j k} \delta_{i l}, \quad\left[\left(B_{a}\right)_{i j},\left(B_{b}^{\dagger}\right)_{k l}\right]=\delta_{a b} \delta_{j k} \delta_{i l} .
$$

Gauge covariant states are obtained by acting with $A^{\dagger}$ and $B^{\dagger}$ on the vacuum with $A|0\rangle=$ $B|0\rangle=0$. Because the gauge invariance enforces all upper indices to be contracted with all lower indices, all gauge invariant operators take the form of $t r_{m, n}\left(\Sigma(b)\left(A^{\dagger}\right)^{\otimes m} \otimes\left(B^{\dagger}\right)^{\otimes n}\right)$. Here $\Sigma$ is a map from elements in $B_{N}(m, n)$ to elements in $\mathbb{C}\left(S_{m+n}\right)$ introduced in [1] 9 . Our claim in the previous sections can be straightforwardly applied to give the following orthogonal state,

$$
=\sum_{m_{A}, m_{A}^{\prime}, m_{\Lambda_{1}}}^{\left|\mathcal{O}_{\Lambda, M_{\Lambda}, \Lambda_{1}, \tau}^{\gamma, A, i j, \tau_{\Lambda_{1}}}\right\rangle} C_{A, m_{A}, A, m_{A}^{\prime}}^{\tau_{\Lambda_{1}, \Lambda_{1}, m_{\Lambda_{1}}}} \sum_{b} D_{A, m_{A}, j, m_{A}^{\prime}, i}^{\gamma}\left(b^{*}\right) \sum_{\vec{a}, \vec{b}} C_{\Lambda, M_{\Lambda}, \Lambda_{1}, m_{\Lambda_{1}, \tau}}^{\vec{a}, \vec{b}} \operatorname{tr}_{m, n}\left(\Sigma(b) O_{\vec{a}, \vec{b}}\right)|0\rangle,
$$

where

$$
O_{\vec{a}, \vec{b}}=A_{a_{1}}^{\dagger} \otimes \cdots \otimes A_{a_{m}}^{\dagger} \otimes B_{b_{1}}^{\dagger} \otimes \cdots \otimes B_{b_{n}}^{\dagger}
$$

The inner product is diagonal:

$$
\left\langle\mathcal{O}_{\Lambda^{\prime}, M_{\Lambda^{\prime}}^{\prime}, \Lambda_{1}^{\prime}, \tau^{\prime}}^{\gamma^{\prime}, A^{\prime}, i^{\prime} j^{\prime}, \tau_{\Lambda^{\prime}}^{\prime}} \mid \mathcal{O}_{\Lambda, M_{\Lambda}, \Lambda_{1}, \tau}^{\gamma, A, i j, \tau_{\Lambda_{1}}}\right\rangle=m ! n ! d_{\Lambda_{1}} \frac{1}{t \gamma} \delta_{\gamma \gamma^{\prime}} \delta_{A A^{\prime}} \delta_{\Lambda \Lambda^{\prime}} \delta_{M_{\Lambda} M_{\Lambda^{\prime}}^{\prime}} \delta_{\Lambda_{1} \Lambda_{1}^{\prime}} \delta_{\tau_{\Lambda_{1}} \tau_{\Lambda_{1}^{\prime}}^{\prime}} \delta_{\tau \tau^{\prime}} \delta_{i i^{\prime}} \delta_{j j^{\prime}}
$$

\footnotetext{
${ }^{9}$ One simple example is $\operatorname{tr}\left(X X^{\dagger}\right)=t r_{1,1}\left(C X \otimes X^{*}\right)=t r_{2}\left(\Sigma(C) X \otimes X^{\dagger}\right)$. The map $\Sigma$ was also exploited in 14 to construct another non-holomorphic extension for a single complex matrix.
} 
Before showing a set of conserved charges whose eigenvalues identify the labels of the orthogonal state, we shall explain symmetries of the Hamiltonian.

The original scalar matrix field theory action is invariant under the adjoint unitary transformation. We can express the conserved matrix current in terms of the annihilation and creation operators as $\left[A_{a}, A_{a}^{\dagger}\right]$ for the $A$-sector and $\left[B_{a}, B_{a}^{\dagger}\right]$ for the $B$-sector. We will find that we have more symmetries at the free level. In order to see enhanced symmetries of this Hamiltonian, we decompose 10 the generator and name them as

$$
\begin{aligned}
\left(G_{L, A}\right)_{i j} & :=\sum_{a}\left(G_{L, A_{a}}\right)_{i j}, \quad\left(G_{L, A_{a}}\right)_{i j}:=\left(A_{a}^{\dagger}\right)_{k j}\left(A_{a}\right)_{i k} \\
\left(G_{R, A}\right)_{i j} & :=\sum_{a}\left(G_{R, A_{a}}\right)_{i j}, \quad\left(G_{R, A_{a}}\right)_{i j}:=\left(A_{a}^{\dagger}\right)_{i k}\left(A_{a}\right)_{k j}
\end{aligned}
$$

and similarly for the $B$-sector. We also define

$$
\begin{aligned}
\left(G_{L}\right)_{i j} & :=\left(G_{L, A}\right)_{i j}+\left(G_{L, B}\right)_{i j}, \\
\left(G_{R}\right)_{i j} & :=\left(G_{R, A}\right)_{i j}+\left(G_{R, B}\right)_{i j} .
\end{aligned}
$$

They generate the left action and the right action:

$$
\begin{aligned}
& {\left[\operatorname{tr}\left(\Lambda G_{L, A}\right),\left(A_{a}\right)_{i j}\right]=-\left(\Lambda A_{a}\right)_{i j}} \\
& {\left[\operatorname{tr}\left(\Lambda G_{R, A}\right),\left(A_{a}\right)_{i j}\right]=\left(A_{a} \Lambda\right)_{i j} .}
\end{aligned}
$$

It is easy to show that

$$
\left[G_{L, A}, \operatorname{tr}\left(A_{a} A_{a}^{\dagger}\right)\right]=0, \quad\left[G_{R, A}, \operatorname{tr}\left(A_{a} A_{a}^{\dagger}\right)\right]=0,
$$

and similar equations for the $B$-sector, implying that all of $G_{L, A}, G_{R, A}$ and $G_{L, B}, G_{R, B}$ generate symmetries of the Hamiltonian. They form the $u(N)$ commutation relations

$$
\begin{aligned}
& {\left[\left(G_{L, A_{a}}\right)_{i j},\left(G_{R, A_{a}}\right)_{k l}\right]=0,} \\
& {\left[\left(G_{L, A_{a}}\right)_{i j},\left(G_{L, A_{b}}\right)_{k l}\right]=\delta_{a b}\left(\left(G_{L, A_{a}}\right)_{k j} \delta_{i l}-\left(G_{L, A_{a}}\right)_{i l} \delta_{j k}\right),} \\
& {\left[\left(G_{R, A_{a}}\right)_{i j},\left(G_{R, A_{b}}\right)_{k l}\right]=\delta_{a b}\left(\left(G_{R, A_{a}}\right)_{i l} \delta_{j k}-\left(G_{R, A_{a}}\right)_{k j} \delta_{i l}\right) .}
\end{aligned}
$$

Since we also have the same relations for the $B$-sector, we thus have four commuting copies of the $u(N)$ algebra.

In terms of these generators, the Hamiltonian and the angular momentum can be expressed by

$$
\begin{aligned}
& H=\operatorname{tr}\left(G_{L, A}+G_{L, B}\right), \\
& J_{a}=\operatorname{tr}\left(G_{L, B_{a}}-G_{L, A_{a}}\right) .
\end{aligned}
$$

It is mentioned that $\operatorname{tr}\left(G_{L, A_{a}}\right)=\operatorname{tr}\left(G_{R, A_{a}}\right)$ and $\operatorname{tr}\left(G_{L, B_{a}}\right)=\operatorname{tr}\left(G_{R, B_{a}}\right)$. Because $G_{L}+G_{R}$ generates the adjoint gauge transformation, we have

$$
G_{L}+G_{R}=0
$$

\footnotetext{
10 Such decomposition was also considered in [24].
} 
on gauge invariant states.

From now on we present some conserved operators which act on the orthogonal state with eigenvalues measuring the representation labels. We shall build operators from the symmetry generators, so it is manifest that they commute with the Hamiltonian. The construction of those operators is shown in [3. We define the symbol $\doteq$ to assume the actions on $\left|\mathcal{O}_{\Lambda, M_{\Lambda}, \Lambda_{1}, \tau}^{\gamma, A, i j, \tau_{\Lambda_{1}}}\right\rangle$. We first show three operators

$$
\begin{aligned}
& \operatorname{tr}\left(G_{L}\right)^{2} \doteq \operatorname{tr}\left(G_{R}\right)^{2} \doteq C_{2}(\gamma), \\
& \operatorname{tr}\left(G_{L, A}\right)^{2} \doteq \operatorname{tr}\left(G_{R, A}\right)^{2} \doteq C_{2}(\alpha), \\
& \operatorname{tr}\left(G_{L, B}\right)^{2} \doteq \operatorname{tr}\left(G_{R, B}\right)^{2} \doteq C_{2}(\beta),
\end{aligned}
$$

where $C_{2}$ is the quadratic Casimir of $U(N)$. These commute each other without assuming the action on the state. We also have similar equations for higher order actions, for example,

$$
\operatorname{tr}\left(G_{L}\right)^{r} \doteq \operatorname{tr}\left(G_{R}\right)^{r} \doteq C_{r}(\gamma)
$$

It was also shown that the multiplicity associated with the decomposition $\gamma \rightarrow A$ of the colour structure can be measured as

$$
\begin{aligned}
& \operatorname{tr}\left(\left(G_{L, A}\right)^{2} G_{L, B}\right) \doteq C(\gamma, \alpha, \beta, i), \\
& \operatorname{tr}\left(\left(G_{R, A}\right)^{2} G_{R, B}\right) \doteq C(\gamma, \alpha, \beta, j),
\end{aligned}
$$

where $C(\gamma, \alpha, \beta, i)$ is a quantity depending on $\gamma, \alpha, \beta, i$, but the exact form has not been found 11 .

In addition to the enhanced symmetry generators $G_{L, A}, G_{R, A}$ and $G_{L, B}, G_{R, B}$, we have another enhanced symmetry which is generated by

$$
\left(G_{E}\right)_{k l j m}:=\left(G_{E, A}\right)_{k l j m}+\left(G_{E, B}\right)_{k l j m},
$$

where

$$
\left(G_{E, A}\right)_{k l j m}:=\sum_{a}\left(G_{E, A_{a}}\right)_{k l j m}:=\sum_{a}\left(A_{a}\right)_{k l}^{\dagger}\left(A_{a}\right)_{j m}
$$

We find that this generates a $U\left(N^{2}\right)$ symmetry, which can be manifested by introducing a composite index $I=(i, j)$, where $I$ takes $N^{2}$ values when $i$ and $j$ run over $N$ values. The use of the composite index enables us to express the generator as

$$
\left(G_{E, A}\right)_{I J}=\sum_{a}\left(A_{a}\right)_{I}^{\dagger}\left(A_{a}\right)_{J}
$$

The commutation relation of $G_{E}$ is

$$
\left[\left(G_{E}\right)_{I J},\left(G_{E}\right)_{K L}\right]=\left(G_{E}\right)_{I L} \delta_{J K}-\left(G_{E}\right)_{K J} \delta_{I L}
$$

\footnotetext{
${ }^{11}$ In the paper [3], it was shown that $\operatorname{tr}\left(\left(G_{L, A}\right)^{2} G_{L, B}\right)$ can recognise the multiplicity index, and it was confirmed for some examples.
} 
The actions of $G_{E, A}$ on $A_{a}$ and $A_{a}^{\dagger}$ are

$$
\begin{aligned}
& {\left[\left(G_{E, A}\right)_{I J},\left(A_{b}^{\dagger}\right)_{K}\right]=\delta_{J K}\left(A_{b}^{\dagger}\right)_{I},} \\
& {\left[\left(G_{E, A}\right)_{I J},\left(A_{b}\right)_{K}\right]=-\delta_{I K}\left(A_{b}\right)_{J},}
\end{aligned}
$$

where we have defined $\delta_{J K}=\delta_{j_{1} k_{2}} \delta_{j_{2} k_{1}}$ for $J=\left(j_{1}, j_{2}\right)$ and $K=\left(k_{1}, k_{2}\right)$. We also have

$$
\begin{aligned}
& {\left[\left(G_{E, A}\right)_{k l j m},\left(G_{L, A}\right)_{p q}\right]=\left(G_{E, A}\right)_{k l p m} \delta_{j q}-\left(G_{E, A}\right)_{k q j m} \delta_{l p},} \\
& {\left[\left(G_{E, A}\right)_{k l j m},\left(G_{R, A}\right)_{p q}\right]=\left(G_{E, A}\right)_{k l j q} \delta_{p m}-\left(G_{E, A}\right)_{p l j m} \delta_{q k} .}
\end{aligned}
$$

It is also easy to see that the symmetry generated by $G_{E}$ is indeed a symmetry of the Hamiltonian:

$$
\left[\left(G_{E, A}\right)_{k l j m}, \operatorname{tr}\left(A_{a}^{\dagger} A_{a}\right)\right]=0 .
$$

With the enhanced $U\left(N^{2}\right)$ symmetry we can define an operator which can measure $\tau_{1}$, i.e. the number of copies of $\Lambda_{1}$ in the inner tensor product of $A \otimes A$, as

$$
\operatorname{tr}\left(G_{L} G_{E} G_{R}\right) \doteq C\left(\tau_{\Lambda_{1}}\right) .
$$

$C\left(\tau_{\Lambda_{1}}\right)$ is a quantity depending on $\tau_{1}$.

The representation labels associated with the flavour indices can be measured in terms of $E_{A a b}:=\operatorname{tr}\left(A_{a}^{\dagger} A_{b}\right)$, whose action on $A^{\dagger}$ is

$$
\left[E_{A a b},\left(A_{c}^{\dagger}\right)_{i j}\right]=\delta_{b c}\left(A_{a}^{\dagger}\right)_{i j} .
$$

$E_{A a b}$ satisfies the $u(p)$ commutation relation:

$$
\left[E_{A a b}, E_{A c d}\right]=\delta_{b c} E_{A a d}-\delta_{a d} E_{A c b} .
$$

The quadratic Casimir of $u(p)$ appears an eigenvalue of the quadratic action of $E_{a b}:=$ $E_{A a b}+E_{B a b}$ on the orthogonal state:

$$
E_{a b} E_{a b} \doteq C_{2}(\Lambda)
$$

We also have

$$
E_{A a b} E_{A a b} \doteq C_{2}\left(\alpha_{1}\right), \quad E_{B a b} E_{B a b} \doteq C_{2}\left(\beta_{1}\right)
$$

for $\Lambda_{1}=\left(\alpha_{1}, \beta_{1}\right)$. The index $\tau$ which runs over the multiplicity of $\Lambda_{1}$ in $\Lambda$ would be measured by

$$
E_{A a b} E_{A b c} E_{B c a} .
$$

In summary, we have provided some conserved operators which can be diagonalised by the orthogonal state. In other words, the orthogonal state proposed in this paper can be specified by the simultaneous diagonalisation of the conserved operators.

Operators which have the diagonal actions on an orthogonal state can be organised by the enhanced symmetries of the Hamiltonian. General multi-trace operators made from those generators are all conserved, and they would form an extension of the $W_{\infty}$ algebra. It is interesting to ask how the enhanced symmetries can be connected with the integrability of this system. Conserved charges may also be helpful to understand gravitational duals or space-time interpretations (see [25, 26] for such discussions). 


\section{Discussions}

In this paper, we have proposed an orthogonal set of non-holomorphic gauge invariant operators made from some complex matrices at the free coupling based on the Brauer algebra. Below are possible future directions along this line.

One remaining problem which should be discussed is to see if this proposed operator exhausts multi-matrix gauge invariant operators. For the case of a single complex matrix, a counting formula in terms of group theoretic languages was given in [1], and it is proved for large $N$ in [27]. It has not, however, been cleared how the counting is modified when $N$ is finite in the context of Brauer algebra. One modification of the finite $N$ case is to impose the constraint $c_{1}\left(\gamma_{+}\right)+c_{1}\left(\gamma_{-}\right) \leq N$, which is naturally expected from the definition of the $U(N)$ group. This constraint seems to hold the attention because it realises a generalisation of the cut-off $c_{1}(R) \leq N$ for the half-BPS case. In the half-BPS case, the cut-off can be translated into the cut-off for the angular momentum of giant gravitons [10]. (Such an effect was originally studied in [28, 29].) The cut-off for the present case would give a constraint for a composite angular momentum of giant gravitons and anti-giant gravitons. For the single complex matrix case, more analyses at finite $N$ will be given in [27]. The counting operators including the finite $N$ case for the non-holomorphic multi-matrix case will be discussed in future publications.

Another basis of non-holomorphic one-matrix gauge invariant operators was built in [14, where the symmetric group plays a role instead of the Brauer algebra. This basis was originally proposed to study excitations of giant gravitons [30, 31, 32, 33]. It will be possible to construct another basis for non-holomorphic multi-matrix operators as an extension of [14. As is discussed in [3], a difference of two bases in a sector can be explained by the fact that a set of Casimirs characterising an orthogonal basis does not commute with another set of Casimirs characterising another orthogonal basis. It would be nice to ask roles of two different bases from the point of view of dual physics.

For the highest weight state in the half-BPS sector, the physics is characterised by a single Young diagram alone. The number and the angular momentum of giant gravitons are encrypted in a single Young diagram. On the other hand, the Brauer algebra brings in two kinds of representation labels, i.e. $\gamma=\left(\gamma_{+}, \gamma_{-}, k\right)$, and $A=(\alpha, \beta)$. Having diagonalised two-point functions at the free level, the representation labels will be directly related to a tensionless string theory. In our previous paper [1], it was conjectured that the operator has a close connection with a system of giant gravitons and anti-giant gravitons. The $k=0$ representation would be naturally related to such a system, where $\gamma_{+}=\alpha$ would read the number and angular momentum of giant gravitons while $\gamma_{-}=\beta$ would read those of anti-giant gravitons. Because $k$ is the number of boxes which are got rid of from $m$ boxes and $n$ anti-boxes, one can anticipate that $k \neq 0$ representations would contain branes and anti-branes with smaller quantum numbers. At $k=m=n$, one may expect closed string excitations without branes and anti-branes. If we include corrections of the t'Hooft coupling, this system may start showing the instability originated from the existence of 
branes and anti-branes. If our operators can really describe physics of the unstable system, it is natural to expect physics of tachyon condensation to be encoded into the representation labels in a way. Fortunately, it is possible to study this system at finite string scale where tachyon has the negative mass squared because this $S O(6)$ sector is closed at one-loop. In this sense, this system will be a good framework to know how the Brauer algebra captures such an interesting unstable system. The operator mixing problem has been reported in 34, 35, 36, 37, 38. On the other hand, the one-loop correction was studied in the language of the representation basis for the $U(2)$ holomorphic sector in [40], where a restricted mixing pattern was found for Young diagrams. A similar restricted mixing was also given in [32, 33 , in the context of the restricted Schur polynomials. The mixing problem was studied in [39] in terms of a basis expressed by the symmetric group, and the Hamiltonian was expressed by splitting and joining interactions. Studies along these lines using the bases proposed in this paper would shed light on the role of the Brauer algebra.

Our orthogonal operators are entirely specified by some group theoretic operators constructed from generators for the enhanced symmetries. Because Casimir operators know information about representation labels of orthogonal sets, studies of Casimirs operators at the one-loop level would tell us about the operator mixing. For example, a relation between the one-loop dilatation operator and the Casimirs may suggest how orthogonal operators mix under quantum corrections. The breaking of the enhanced symmetries with the interactions turned on can be possibly associated with the operator mixing problem. We hope to understand the role of the enhanced symmetries by looking for a connection to the integrability.

\section{Acknowledgements}

I would like to thank Sanjaye Ramgoolam for valuable discussions and for collaboration at the initial stage of this work, and Tom Brown, Robert de Mello Koch, Paul Heslop, David Turton for useful discussions. I gratefully acknowledge the support of STFC grant PP/D507323/1. I also thank Okayama Institute for Quantum Physics for hospitality, where

the final part of this work was done. Special thanks to Sea the Stars and Liverpool FC for giving me encouragement.

\section{A One-loop dilatation operator}

In this section, we rewrite the one-loop dilatation operator in terms of the complex variables. The dilatation operator in the $S O(6)$ sector up to one-loop order was given [41, 42] by

$$
\mathcal{D}=\mathcal{D}_{0}+\mathcal{D}_{2}:=\mathcal{D}_{0}+g^{2} \mathcal{H}, \quad\left(g^{2}=g_{Y M}^{2} N / 8 \pi^{2}\right)
$$


where

$$
\mathcal{H}=N^{-1}\left(-\frac{1}{2}: \operatorname{tr}\left[\Phi_{m}, \Phi_{n}\right]\left[\check{\Phi}^{m}, \check{\Phi}^{n}\right]:-\frac{1}{4}: \operatorname{tr}\left[\Phi_{m}, \check{\Phi}^{n}\right]\left[\Phi_{m}, \check{\Phi}^{n}\right]:\right)
$$

$\Phi_{m}(m=1, \ldots, 6)$ is the scalar fields. $\check{\Phi}$ represents the following derivative action

$$
\left(\check{\Phi}^{m}\right)_{i j}\left(\Phi_{n}\right)_{k l}=\delta_{n}^{m} \delta_{i l} \delta_{k j}
$$

for the $u(N)$ gauge group.

We define the complex combination of two scalars

$$
X_{a}=\Phi_{2 a-1}+i \Phi_{2 a}, \quad(a=1,2,3) .
$$

Solving for $\Phi$, we get

$$
\Phi_{2 a-1}=\frac{1}{2}\left(X_{a}+X_{a}^{\dagger}\right), \quad \Phi_{2 a}=\frac{1}{2 i}\left(X_{a}-X_{a}^{\dagger}\right) .
$$

We express these equations for later convenience as

$$
\Phi_{m}=a_{m}^{i} Z_{i}
$$

where $Z_{i}=\left(X_{1}, X_{1}^{\dagger}, X_{2}, X_{2}^{\dagger}, X_{3}, X_{3}^{\dagger}\right)$. The derivative of the complex matrix is defined by

$$
\check{X}^{a}=\frac{1}{2}\left(\check{\Phi}^{2 a-1}-i \check{\Phi}^{2 a}\right)
$$

satisfying $\left(\check{X}^{a}\right)_{i j}\left(X_{b}\right)_{k l}=\delta_{a b} \delta_{i l} \delta_{k l}$. We solve for $\check{\Phi}$ as

$$
\check{\Phi}_{2 a-1}=\check{X}_{a}+\check{X}_{a}^{\dagger}, \quad \check{\Phi}_{2 a}=i\left(\check{X}_{a}-\check{X}_{a}^{\dagger}\right),
$$

which we shall denote compactly by

$$
\check{\Phi}_{m}=b_{m}^{i} \check{Z}_{i},
$$

where $\check{Z}_{i}=\left(\check{X}_{1}, \check{X}_{1}^{\dagger}, \check{X}_{2}, \check{X}_{2}^{\dagger}, \check{X}_{3}, \check{X}_{3}^{\dagger}\right)$.

Using these complex variables, we can rewrite the first term of the Hamiltonian (A.2) as

$$
\begin{aligned}
\operatorname{tr}\left[\Phi_{m}, \Phi_{n}\right]\left[\check{\Phi}^{m}, \check{\Phi}^{n}\right]= & a_{m}^{i} a_{n}^{j} b_{m}^{k} b_{n}^{l} \operatorname{tr}\left[Z_{i}, Z_{j}\right]\left[\check{Z}^{k}, \check{Z}^{l}\right] \\
= & g^{i k} g^{j l} \operatorname{tr}\left[Z_{i}, Z_{j}\right]\left[\check{Z}^{k}, \check{Z}^{l}\right] \\
= & \operatorname{tr}\left[X_{a}, X_{b}\right]\left[\check{X}_{a}, \check{X}_{b}\right]+\operatorname{tr}\left[X_{a}, X_{b}^{\dagger}\right]\left[\check{X}_{a}, \check{X}_{b}^{\dagger}\right] \\
& +\operatorname{tr}\left[X_{a}^{\dagger}, X_{b}\right]\left[\check{X}_{a}^{\dagger}, \check{X}_{b}\right]+\operatorname{tr}\left[X_{a}^{\dagger}, X_{b}^{\dagger}\right]\left[\check{X}_{a}^{\dagger}, \check{X}_{b}^{\dagger}\right]
\end{aligned}
$$

We have defined the metric $g^{i k}=\sum_{m} a_{m}^{i} b_{m}^{k}=\delta^{i k}$. On the other hand, the second term of the Hamiltonian can be rewritten as

$$
\operatorname{tr}\left[\Phi_{m}, \check{\Phi}^{n}\right]\left[\Phi_{m}, \check{\Phi}^{n}\right]=a_{m}^{i} b_{n}^{j} a_{m}^{k} b_{n}^{l} \operatorname{tr}\left[Z_{i}, \check{Z}^{j}\right]\left[Z_{k}, \check{Z}^{l}\right]
$$




$$
\begin{aligned}
= & h^{i k} \tilde{h}^{j l} \operatorname{tr}\left[Z_{i}, \check{Z}^{j}\right]\left[Z_{k}, \check{Z}^{l}\right] \\
= & \operatorname{tr}\left[X_{a}, \check{X}_{b}\right]\left[X_{a}^{\dagger}, \check{X}_{b}^{\dagger}\right]+\operatorname{tr}\left[X_{a}, \check{X}_{b}^{\dagger}\right]\left[X_{a}^{\dagger}, \check{X}_{b}\right] \\
& +\operatorname{tr}\left[X_{a}^{\dagger}, \check{X}_{b}^{\dagger}\right]\left[X_{a}, \check{X}_{b}\right]+\operatorname{tr}\left[X_{a}^{\dagger}, \check{X}_{b}\right]\left[X_{a}, \check{X}_{b}^{\dagger}\right] \\
= & 2 \operatorname{tr}\left[X_{a}, \check{X}_{b}\right]\left[X_{a}^{\dagger}, \check{X}_{b}^{\dagger}\right]+2 \operatorname{tr}\left[X_{a}, \check{X}_{b}^{\dagger}\right]\left[X_{a}^{\dagger}, \check{X}_{b}\right]
\end{aligned}
$$

where we have defined the metric as $h^{i k}=a_{m}^{i} a_{m}^{k}$ and $\tilde{h}^{j l}=b_{n}^{j} b_{n}^{l}$, with non-zero components $h^{X_{a} \bar{X}_{a}}=1 / 2$ and $\tilde{h}^{X_{a} \bar{X}_{a}}=2$. Collecting (A.10) and (A.11), we get the Hamiltonian in terms of the complex variables as

$$
\begin{aligned}
\mathcal{H}= & -\frac{1}{2 N}:\left(\operatorname{tr}\left[X_{a}, X_{b}\right]\left[\check{X}_{a}, \check{X}_{b}\right]+\operatorname{tr}\left[X_{a}, X_{b}^{\dagger}\right]\left[\check{X}_{a}, \check{X}_{b}^{\dagger}\right]\right. \\
& +\operatorname{tr}\left[X_{a}^{\dagger}, X_{b}\right]\left[\check{X}_{a}^{\dagger}, \check{X}_{b}\right]+\operatorname{tr}\left[X_{a}^{\dagger}, X_{b}^{\dagger}\right]\left[\check{X}_{a}^{\dagger}, \check{X}_{b}^{\dagger}\right] \\
& \left.+\operatorname{tr}\left[X_{a}, \check{X}_{b}\right]\left[X_{a}^{\dagger}, \check{X}_{a}^{\dagger}\right]+\operatorname{tr}\left[X_{a}, \check{X}_{b}^{\dagger}\right]\left[X_{a}^{\dagger}, \check{X}_{b}\right]\right):
\end{aligned}
$$

\section{B Proof of the diagonal two-point function}

In this section, we show detailed calculations of the two-point functions.

\section{B.1 Two-point function for the covariant operator}

The two-point function of the representation basis (22) can be computed as

$$
\begin{aligned}
& \left\langle:\left(O_{\Lambda, M_{\Lambda}, \Lambda_{1}, m_{\Lambda_{1}, \tau}}^{\dagger}\right)_{J}^{I}::\left(O_{\Lambda^{\prime}, M_{\Lambda^{\prime}}^{\prime}, \Lambda_{1}^{\prime}, m_{\Lambda_{1}^{\prime}}^{\prime}, \tau^{\prime}}\right)_{L}^{K}:\right\rangle \\
& =\sum_{a, b, a^{\prime}, b^{\prime}}\left(C_{\Lambda, M_{\Lambda}, \Lambda_{1}, m_{\Lambda_{1}, \tau}}^{\vec{a}, \vec{b}}\right)^{*} C_{\Lambda^{\prime}, M_{\Lambda^{\prime}}^{\prime}, \Lambda_{1}^{\prime}, m_{\Lambda_{1}^{\prime}}^{\prime}, \tau^{\prime}}^{a^{\prime}}\left\langle:\left(O_{\vec{a}, \vec{b}}^{\dagger}\right)_{J}^{I}::\left(O_{\overrightarrow{a^{\prime}, b^{\prime}}}\right)_{L}^{K}:\right\rangle \\
& =\sum_{a, b, a^{\prime}, b^{\prime}}\left(C_{\Lambda, M_{\Lambda}, \Lambda_{1}, m_{\Lambda_{1}}, \tau}^{\vec{a}, \vec{b}}\right)^{*} C_{\Lambda^{\prime}, M_{\Lambda^{\prime}}}^{\overrightarrow{a^{\prime}}, \vec{b}_{1}^{\prime}, m_{\Lambda_{1}^{\prime}}^{\prime}, \tau^{\prime}} \sum_{\sigma \in S_{m} \times S_{n}} \prod_{k=1}^{m} \delta_{a_{k} a_{\sigma(k)}^{\prime}} \prod_{l=1}^{n} \delta_{b_{l} b_{\sigma(l)}^{\prime}}(\sigma)_{J}^{K}\left(\sigma^{-1}\right)_{L}^{I} \\
& =\sum_{a, b} \sum_{\sigma \in S_{m} \times S_{n}}\left(C_{\Lambda, M_{\Lambda}, \Lambda_{1}, m_{\Lambda_{1}}, \tau}^{\vec{a}, \vec{b}}\right)^{*} C_{\Lambda^{\prime}, M_{\Lambda^{\prime}}^{\prime}, \Lambda_{1}^{\prime}, m_{\Lambda_{1}^{\prime}}^{\prime}, \tau^{\prime}}^{a_{\sigma^{\prime}}{ }^{\prime}(1), \cdots, a_{\sigma^{-1}}, \cdots, b_{\sigma^{-1}(n)}}(\sigma)_{J}^{K}\left(\sigma^{-1}\right)_{L}^{I} \\
& =\delta_{\Lambda \Lambda^{\prime}} \delta_{M_{\Lambda} M_{\Lambda^{\prime}}^{\prime}} \delta_{\Lambda_{1} \Lambda_{1}^{\prime}} \delta_{\tau \tau^{\prime}} \sum_{\sigma \in S_{m} \times S_{n}} D_{m_{\Lambda_{1}} m_{\Lambda_{1}^{\prime}}^{\prime}}^{\Lambda_{1}}(\sigma)(\sigma)_{J}^{K}\left(\sigma^{-1}\right)_{L}^{I} .
\end{aligned}
$$

To get the third equality, we solved the delta symbols. In the last step, we have used

$$
C_{\Lambda^{\prime}, M_{\Lambda^{\prime}}^{\prime}, \Lambda_{1}^{\prime}, m_{\Lambda_{1}^{\prime}}^{\prime}, \tau^{\prime}}^{a_{\sigma^{-1}(1)}, \cdots, a_{\sigma^{-1}(1)}, \cdots, b_{\sigma^{-1}(n)}}=D_{m_{\Lambda_{1}^{\prime}}^{\prime} m_{\Lambda_{1}^{\prime \prime}}^{\Lambda_{1}^{\prime}}}^{\Lambda^{\prime}}\left(\sigma^{-1}\right) C_{\Lambda^{\prime}, M_{\Lambda^{\prime}}^{\prime}, \Lambda_{1}^{\prime}, m_{\Lambda_{1}^{\prime}}^{\prime \prime}, \tau^{\prime}}^{a_{1}, \cdots, a_{m}, b_{1}, \cdots, b_{n}}
$$

and the following equation

$$
\sum_{\vec{a}, \vec{b}}\left(C_{\vec{a}, \vec{b}}^{\Lambda, M_{\Lambda}, \Lambda_{1}, m_{\Lambda_{1}}, \tau}\right)^{*} C_{\vec{a}, \vec{b}}^{\Lambda^{\prime}, M_{\Lambda^{\prime}}^{\prime}, \Lambda_{1}^{\prime}, m_{\Lambda_{1}^{\prime}}^{\prime}, \tau^{\prime}}=\delta_{\Lambda \Lambda^{\prime}} \delta_{M_{\Lambda} M_{\Lambda^{\prime}}^{\prime}} \delta_{\Lambda_{1} \Lambda_{1}^{\prime}} \delta_{m_{\Lambda_{1}} m_{\Lambda_{1}^{\prime}}^{\prime}} \delta_{\tau \tau^{\prime}}
$$

The derivation of this equation is completely similar to (69) in [17]. (B.2) comes from the fact that $\sigma^{-1}$ acts only on $V_{\Lambda_{1}}^{\mathbb{C}\left(S_{m} \times S_{n}\right)}$ when it acts on $V^{\otimes m} \otimes \bar{V}^{\otimes n}$ in (20). 


\section{B.2 Two-point function for the gauge invariant operator}

In this subsection, we shall present the proof of (31). Using the two-point function of the covariant operator (26), we get

$$
\begin{aligned}
&\left\langle\mathcal{O}_{\Lambda, M_{\Lambda}, \Lambda_{1}, \tau}^{\gamma, A, i j, \tau_{\Lambda_{1}} \dagger} \mathcal{O}_{\Lambda^{\prime}, M_{\Lambda^{\prime}}^{\prime}, \Lambda_{1}^{\prime}, \tau^{\prime}}^{\gamma^{\prime}, A^{\prime}, i^{\prime} j^{\prime}, \tau_{\Lambda^{\prime}}^{\prime}}\right\rangle \\
&= \delta_{\Lambda \Lambda^{\prime}} \delta_{M_{\Lambda} M_{\Lambda^{\prime}}^{\prime}} \delta_{\Lambda_{1} \Lambda_{1}^{\prime}} \delta_{\tau \tau^{\prime}} C_{A, m_{A}, A, m_{A}^{\prime}}^{\tau_{\Lambda_{1}}, \Lambda_{1}, m_{\Lambda_{1}}} C_{A_{1}^{\prime}, m_{A^{\prime}}, A^{\prime}, m_{A^{\prime}}^{\prime}}^{\tau_{\Lambda^{\prime}}^{\prime}, \Lambda_{1}^{\prime}, m_{\Lambda^{\prime}}^{\prime}} \\
& \sum_{\sigma \in S_{m} \times S_{n}} D_{m_{\Lambda_{1}} m_{\Lambda_{1}^{\prime}}^{\prime}}^{\Lambda_{1}^{\prime}}(\sigma) \sum_{b, b^{\prime} \in B_{N}(m, n)} D_{A, m_{A} j, m_{A}^{\prime} i}^{\gamma}\left(b^{*}\right) D_{A^{\prime}, m_{A^{\prime}} j^{\prime}, m_{A^{\prime}}^{\prime} i^{\prime}}^{\gamma^{\prime}} \\
&\left.b^{\prime *}\right) t r_{m, n}\left(b^{\dagger} \sigma b^{\prime} \sigma^{-1}\right),(B)
\end{aligned}
$$

where we have used the fact that the Clebsch-Gordan coefficient $C_{A, m_{A}, A, m_{A}^{\prime}}^{\tau_{\Lambda_{1}}, \Lambda_{1}, m_{\Lambda_{1}}}$ and the representation matrix of Brauer elements are real.

Let us work on $\operatorname{tr}_{m, n}\left(b \sigma b^{\prime} \sigma^{-1}\right)$. Using the Schur-Weyl duality ([6), we find

$$
t r_{m, n}\left(b^{\dagger} \sigma b^{\prime} \sigma^{-1}\right)=\sum_{\gamma^{\prime \prime}} t^{\gamma^{\prime \prime}} \chi^{\gamma^{\prime \prime}}\left(b^{\dagger} \sigma b^{\prime} \sigma^{-1}\right)
$$

where $t^{\gamma^{\prime \prime}}$ and $\chi^{\gamma^{\prime \prime}}(b)$ are the dimension of $U(N)$ and the character of the Brauer algebra associated with the irreducible representation $\gamma^{\prime \prime}$. We decompose the character by inserting the completeness of $\left|\gamma \rightarrow A, m_{A}, i_{A}\right\rangle$ as

$$
\begin{aligned}
\chi^{\gamma^{\prime \prime}}\left(b^{\dagger} \sigma b^{\prime} \sigma^{-1}\right)= & \left\langle\gamma^{\prime \prime} \rightarrow B, m_{B}, i_{B}\left|b^{\dagger}\right| \gamma^{\prime \prime} \rightarrow C, m_{C}, i_{C}\right\rangle D_{m_{C} m_{C}^{\prime}}^{C}(\sigma) \\
& \left\langle\gamma^{\prime \prime} \rightarrow C, m_{C}^{\prime}, i_{C}\left|b^{\prime}\right| \gamma^{\prime \prime} \rightarrow B, m_{B}^{\prime}, i_{B}\right\rangle D_{m_{B}^{\prime} m_{B}}^{B}\left(\sigma^{-1}\right),
\end{aligned}
$$

where the summation is assumed to be taken for all repeated letters except $\gamma^{\prime \prime}$. We note that

$$
\left\langle\gamma^{\prime \prime} \rightarrow B, m_{B}, i_{B}|\sigma| \gamma^{\prime \prime} \rightarrow C, m_{C}, i_{C}\right\rangle=D_{m_{B} m_{C}}^{B}(\sigma) \delta_{B C} \delta_{i_{B} i_{C}}
$$

Using the above decomposition of the character, one can calculate (B.4) as

$$
\begin{aligned}
& \left\langle\mathcal{O}_{\Lambda, M_{\Lambda}, \Lambda_{1}, \tau}^{\gamma, A, i j, \tau_{\Lambda_{1}} \dagger} \mathcal{O}_{\Lambda^{\prime}, M_{\Lambda^{\prime}}^{\prime}, \Lambda_{1}^{\prime}, \tau^{\prime}}^{\gamma^{\prime}, A^{\prime}, i^{\prime} j^{\prime}, \tau_{\Lambda^{\prime}}^{\prime}}\right\rangle \\
& =\delta_{\Lambda \Lambda^{\prime}} \delta_{M_{\Lambda} M_{\Lambda^{\prime}}^{\prime}} \delta_{\Lambda_{1} \Lambda_{1}^{\prime}} \delta_{\tau \tau^{\prime}} C_{A, m_{A}, A, m_{A}^{\prime}}^{\tau_{\Lambda_{1}, \Lambda_{1}, m_{\Lambda_{1}}}} C_{A^{\prime}, m_{A^{\prime}}, A^{\prime}, m_{A^{\prime}}^{\prime}}^{\tau_{\Lambda_{1}^{\prime}, \Lambda_{1}^{\prime}, m^{\prime}}^{\prime}} \\
& \sum_{\sigma \in S_{m} \times S_{n}} D_{m_{\Lambda_{1}} m_{\Lambda_{1}^{\prime}}^{\prime}}^{\Lambda_{1}}(\sigma) \sum_{b, b^{\prime}} D_{A, m_{A} j, m_{A}^{\prime} i}^{\gamma}\left(b^{*}\right) D_{A^{\prime}, m_{A^{\prime} j^{\prime}, m_{A^{\prime}}^{\prime} i^{\prime}}^{\gamma^{\prime}}}^{\gamma}\left(b^{\prime *}\right) \\
& t^{\gamma^{\prime \prime}}\left\langle\gamma^{\prime \prime} \rightarrow B, m_{B}, i_{B}\left|b^{\dagger}\right| \gamma^{\prime \prime} \rightarrow C, m_{C}, i_{C}\right\rangle D_{m_{C} m_{C}^{\prime}}^{C}(\sigma) \\
& \left\langle\gamma^{\prime \prime} \rightarrow C, m_{C}^{\prime}, i_{C}\left|b^{\prime}\right| \gamma^{\prime \prime} \rightarrow B, m_{B}^{\prime}, i_{B}\right\rangle D_{m_{B}^{\prime} m_{B}}^{B}\left(\sigma^{-1}\right) \\
& =\delta_{\Lambda \Lambda^{\prime}} \delta_{M_{\Lambda} M_{\Lambda^{\prime}}^{\prime}} \delta_{\Lambda_{1} \Lambda_{1}^{\prime}} \delta_{\tau \tau^{\prime}} C_{A, m_{A}, A, m_{A}^{\prime}}^{\tau_{\Lambda_{1}, \Lambda_{1}, m_{\Lambda_{1}}}} C_{A^{\prime}, m_{A^{\prime}}, A^{\prime}, m_{A^{\prime}}^{\prime}}^{\tau_{\Lambda^{\prime}}^{\prime}, \Lambda_{1}^{\prime}, m_{B}^{\prime}} \\
& \frac{1}{t^{\gamma}} \delta_{\gamma \gamma^{\prime}} \delta_{A A^{\prime}} \sum_{\sigma \in S_{m} \times S_{n}} D_{m_{\Lambda_{1}} m_{\Lambda_{1}^{\prime}}^{\prime}}^{\Lambda_{1}}(\sigma) D_{m_{A}^{\prime} m_{A^{\prime}}^{\prime}}^{A}(\sigma) D_{m_{A^{\prime}} m_{A}}^{A}\left(\sigma^{-1}\right) \delta_{i^{\prime} i} \delta_{j j^{\prime}}
\end{aligned}
$$




$$
\begin{aligned}
= & \delta_{\Lambda \Lambda^{\prime}} \delta_{M_{\Lambda} M_{\Lambda^{\prime}}^{\prime}} \delta_{\Lambda_{1} \Lambda_{1}^{\prime}} \delta_{\tau \tau^{\prime}} \delta_{A A^{\prime}} C_{A, m_{A}, A, m_{A}^{\prime}}^{\tau_{\Lambda_{1}}, \Lambda_{1}, m_{\Lambda_{1}}} C_{A^{\prime}, m_{A^{\prime}}, A^{\prime}, m_{A^{\prime}}^{\prime}}^{\tau_{\Lambda^{\prime}}^{\prime}, \Lambda_{1}^{\prime}, m^{\prime}}{ }^{\prime} \\
& \frac{1}{t \gamma} \delta_{\gamma \gamma^{\prime}} \frac{m ! n !}{d_{\Lambda_{1}}} \sum_{\tau_{\Lambda_{1}}^{\prime}} \delta_{i i^{\prime}} \delta_{j j^{\prime}} C_{A, m_{A}^{\prime}, A, m_{A}}^{\tau_{\Lambda_{1}}^{\prime}, \Lambda_{1}, m_{\Lambda_{1}}} C_{A, m_{A^{\prime}}^{\prime}, A, m_{A^{\prime}}, m_{\Lambda_{1}^{\prime}}^{\prime}}^{\tau^{\prime}} \\
= & \delta_{\Lambda \Lambda^{\prime}} \delta_{M_{\Lambda} M_{\Lambda^{\prime}}^{\prime}} \delta_{\Lambda_{1} \Lambda_{1}^{\prime}} \delta_{\tau \tau^{\prime}} \delta_{A A^{\prime}} \delta_{\tau_{\Lambda_{1}} \tau_{\Lambda_{1}^{\prime}}^{\prime}} \delta_{i i^{\prime}} \delta_{j j^{\prime}} m ! n ! d_{\Lambda_{1}} \frac{1}{t \gamma} \delta_{\gamma \gamma^{\prime}} .
\end{aligned}
$$

The second equality was obtained by performing the summation for $b$ and $b^{\prime}$ using the formula derived in subsection B.3. To show the third equality, we have used

$$
\begin{aligned}
& \sum_{\sigma \in S_{m} \times S_{n}} D_{m_{\Lambda_{1}} m_{\Lambda_{1}^{\prime}}^{\prime}}^{\Lambda_{1}}(\sigma) D_{m_{A}^{\prime} m_{A^{\prime}}^{\prime}}^{A}(\sigma) D_{m_{A^{\prime}} m_{A}}^{A}\left(\sigma^{-1}\right) \\
= & \sum_{\sigma \in S_{m} \times S_{n}}^{A} D_{m_{\Lambda_{1}} m_{\Lambda_{1}^{\prime}}^{\prime}}^{\Lambda_{1}}(\sigma) D_{m_{A}^{\prime} m_{A^{\prime}}^{\prime}}^{A}(\sigma) D_{m_{A} m_{A^{\prime}}}^{A}(\sigma) \\
= & \frac{m ! n !}{d_{\Lambda_{1}}} \sum_{\tau_{\Lambda_{1}}^{\prime}} C_{A, m_{A}^{\prime}, A, m_{A}}^{\tau_{\Lambda_{1}}^{\prime}, \Lambda_{1}, m_{\Lambda_{1}}} C_{A, m_{A^{\prime}}^{\prime}, A, m_{A^{\prime}}}^{\tau_{\Lambda_{1}}^{\prime}, \Lambda_{1}, m_{\Lambda^{\prime}}^{\prime}}
\end{aligned}
$$

This is a generalisation of (163) in [13]. The forth equality of (B.8) follows from the orthogonality of Clebsch-Gordan coefficient [23]:

$$
\sum_{m_{A}, m_{A}^{\prime}} C_{A, m_{A}, A, m_{A}^{\prime}}^{\tau_{\Lambda_{1}}, \Lambda_{1}, m_{\Lambda_{1}}} C_{A, m_{A}, A, m_{A}^{\prime}}^{\tau_{\Lambda_{1}^{\prime}}, \Lambda_{1}^{\prime}, m_{\Lambda_{1}^{\prime}}}=\delta_{\tau_{\Lambda_{1}, \tau_{\Lambda_{1}^{\prime}}}} \delta_{\Lambda_{1}, \Lambda_{1}^{\prime}} \delta_{m_{\Lambda_{1}}, m_{\Lambda_{1}^{\prime}}}
$$

\section{B.3 A formula}

We now calculate

$$
\sum_{b \in B_{N}(m, n)}\left\langle\gamma \rightarrow A, m_{A}, i_{A}\left|b^{*}\right| \gamma \rightarrow B, m_{B}, i_{B}\right\rangle\left\langle\gamma^{\prime} \rightarrow C, m_{C}, i_{C}|b| \gamma^{\prime} \rightarrow D, m_{D}, i_{D}\right\rangle .
$$

This can be worked out as

$$
\begin{aligned}
& \sum_{b \in B_{N}(m, n)} \sum_{I, J, K, L} B_{\gamma I ; A, m_{A}, i_{A}}^{\dagger} B_{\gamma J ; B, m_{B}, i_{B}} D_{I J}^{\gamma}\left(b^{*}\right) B_{\gamma^{\prime} K ; C, m_{C}, i_{C}}^{\dagger} B_{\gamma^{\prime} L ; D, m_{D}, i_{D}} D_{K L}^{\gamma^{\prime}}(b) \\
= & \frac{1}{t^{\gamma}} \delta_{\gamma \gamma^{\prime}} \sum_{I, J} B_{\gamma I ; A, m_{A}, i_{A}}^{\dagger} B_{\gamma J ; B, m_{B}, i_{B}} B_{\gamma J ; C, m_{C}, i_{C}}^{\dagger} B_{\gamma I ; D, m_{D}, i_{D}} \\
= & \frac{1}{t^{\gamma}} \delta_{\gamma \gamma^{\prime}} \delta_{A D} \delta_{B C} \delta_{m_{A} m_{D}} \delta_{m_{B} m_{C}} \delta_{i_{A} i_{D}} \delta_{i_{B} i_{C}} .
\end{aligned}
$$

Some equations needed to show this are collected in the following.

- A representation matrix for an element $b$ in the Brauer algebra can be denoted by $D_{I J}^{\gamma}(b)=\langle\gamma, I|b| \gamma, j\rangle\left(1 \leq I, J \leq d_{\gamma}\right) . \quad d_{\gamma}$ is the dimension of the Brauer algebra relevant to the irreducible representation $\gamma$. The basis is complete:

$$
\sum_{I=1}^{d_{\gamma}}|\gamma, I\rangle\langle\gamma, I|=1
$$


The orthogonality of the representation matrix is [43]

$$
\sum_{b \in B_{N}(m, n)} D_{I J}^{\gamma}\left(b^{*}\right) D_{K L}^{\gamma^{\prime}}(b)=\frac{1}{t^{\gamma}} \delta_{J K} \delta_{I L} \delta^{\gamma \gamma^{\prime}} .
$$

- We introduce the branching coefficient $B_{\gamma I ; A, m_{A}, i}$ as

$$
B_{\gamma I ; A, m_{A}, i}=\left\langle\gamma, I \mid \gamma \rightarrow A, m_{A}, i\right\rangle .
$$

The orthogonality of the branching coefficient can be derived as

$$
\begin{aligned}
\sum_{I}\left(B_{\gamma, I ; A, m_{A}, i_{A}}\right)^{\dagger} B_{\gamma, I ; B, m_{B}, i_{B}} & =\sum_{I}\left\langle\gamma \rightarrow A, m_{A}, i_{A} \mid \gamma, I\right\rangle\left\langle\gamma, I \mid \gamma \rightarrow B, m_{B}, i_{B}\right\rangle \\
& =\delta_{A B} \delta_{m_{A} m_{B}} \delta_{i_{A} i_{B}},
\end{aligned}
$$

where we have chosen an orthogonal basis

$$
\left\langle\gamma \rightarrow B, m_{B}, k \mid \gamma \rightarrow A, m_{A}, j\right\rangle=\delta_{A B} \delta_{m_{A} m_{B}} \delta_{j k}
$$

\section{Two actions of the symmetric group}

We introduced two Brauer algebras to label the orthogonal basis. One was responsible for flavour indices and the other was for colour indices. They have the same sub-algebra, i.e. the group algebra of the symmetric group $S_{m} \times S_{n}$, but they allow the sub-algebra to act on the operator in different ways. The two different ways are correlated as we shall see in (C.3). Because the orthogonal basis should respect it, the Clebsh-Gordan coefficient should also satisfy a relation. We shall see that this is the case.

The symmetric group associated with colour indices act on the covariant operator as

$$
\begin{aligned}
\sigma\left(O_{\Lambda, M_{\Lambda}, \Lambda_{1}, m_{\Lambda_{1}}, \tau}\right)_{J}^{I} \sigma^{-1} & =\left(O_{\Lambda, M_{\Lambda}, \Lambda_{1}, m_{\Lambda_{1}}, \tau}\right)_{\sigma(J)}^{\sigma(I)} \\
& =\sum_{\vec{a}, \vec{b}} C_{\Lambda, M_{\Lambda}, \Lambda_{1}, m_{\Lambda_{1}}, \tau}^{\vec{a}, \vec{b}}\left(O_{\vec{a}, \vec{b}}\right)_{\sigma(J)}^{\sigma(I)} .
\end{aligned}
$$

On the other hand, the other symmetric group associated with flavour indices act on the covariant operator as

$$
\begin{aligned}
D_{m_{\Lambda_{1}} m_{\Lambda_{1}}^{\prime}(\sigma) O_{\Lambda, M_{\Lambda}, \Lambda_{1}, m_{\Lambda_{1}}^{\prime}, \tau}}^{\Lambda_{1}} & =\sum_{\vec{a}, \vec{b}} C_{\Lambda, M_{\Lambda}, \Lambda_{1}, m_{\Lambda_{1}}, \tau}^{\sigma(\vec{a}), \sigma(\vec{b})}\left(O_{\vec{a}, \vec{b}}\right) \\
& =\sum_{\vec{a}, \vec{b}} C_{\Lambda, M_{\Lambda}, \Lambda_{1}, m_{\Lambda_{1}}, \tau}^{\vec{a}, \vec{b}}\left(O_{\sigma^{-1}(\vec{a}), \sigma^{-1}(\vec{b})}\right) .
\end{aligned}
$$

These two actions are not independent because of

$$
\left(O_{\vec{a}, \vec{b}}\right)_{\sigma(J)}^{\sigma(I)}=\left(O_{\sigma(\vec{a}), \sigma(\vec{b})}\right)_{J}^{I} .
$$


This means the following equation to the covariant operator

$$
O_{\Lambda, M_{\Lambda}, \Lambda_{1}, m_{\Lambda_{1}}, \tau}=D_{m_{\Lambda_{1}} m_{\Lambda_{1}}^{\prime}}^{\Lambda_{1}}(\sigma) \sigma\left(O_{\Lambda, M_{\Lambda}, \Lambda_{1}, m_{\Lambda_{1}}^{\prime}, \tau}\right) \sigma^{-1}
$$

Substituting this in the gauge invariant operator (28), we obtain

$$
\begin{aligned}
\mathcal{O}_{\Lambda, M_{\Lambda}, \Lambda_{1}, \tau}^{\gamma, A, i j, \tau_{1}} & =C_{A, m_{A}, A, m_{A}^{\prime}}^{\tau_{\Lambda_{1}, \Lambda_{1}, m_{\Lambda_{1}}}} \sum_{b} D_{A, m_{A} i, m_{A}^{\prime} j}^{\gamma}\left(b^{*}\right) D_{m_{\Lambda_{1}} m_{\Lambda_{1}}^{\prime}}^{\Lambda_{1}}(\sigma) \operatorname{tr}_{m, n}\left(b \sigma O_{\Lambda, M_{\Lambda}, \Lambda_{1}, m_{\Lambda_{1}}^{\prime}, \tau} \sigma^{-1}\right) \\
& \left.=C_{A, m_{A}, A, m_{A}^{\prime}}^{\tau_{\Lambda_{1}, \Lambda_{1}, m_{\Lambda_{1}}}} \sum_{b} D_{A, m_{A} i, m_{A}^{\prime} j}^{\gamma}\left(\left(\sigma b \sigma^{-1}\right)^{*}\right) D_{m_{\Lambda_{1}} m_{\Lambda_{1}}^{\prime}}^{\Lambda_{1}}(\sigma) \operatorname{tr} m, n, b O_{\Lambda, M_{\Lambda}, \Lambda_{1}, m_{\Lambda_{1}}^{\prime}, \tau}\right) .
\end{aligned}
$$

This $\sigma$-dependence can be shown to vanish as expected from the consistency of this operator. Using 12

$$
\left(\sigma b \sigma^{-1}\right)^{*}=\sigma b^{*} \sigma^{-1}
$$

we can show that the $\sigma$-dependence disappears as

$$
\begin{aligned}
& C_{A, m_{A}, A, m_{A}^{\prime}}^{\tau_{\Lambda_{1}}, \Lambda_{1}, m_{\Lambda_{1}}} \sum_{b} D_{A, m_{A} i, m_{A}^{\prime} j}^{\gamma}\left(\left(\sigma b \sigma^{-1}\right)^{*}\right) D_{m_{\Lambda_{1}} m_{\Lambda_{1}}^{\prime}}^{\Lambda_{1}}(\sigma) \\
= & C_{A, m_{A}, A, m_{A}^{\prime}}^{\tau_{\Lambda_{1}}, \Lambda_{1}, m_{\Lambda_{1}}} \sum_{b} D_{m_{A} m_{A}^{\prime \prime}}^{A}(\sigma) D_{A, m_{A}^{\prime \prime} i, m_{A}^{\prime \prime \prime} j}^{\gamma}\left(b^{*}\right) D_{m_{A}^{\prime \prime \prime} m_{A}^{\prime}}^{A}\left(\sigma^{-1}\right) D_{m_{\Lambda_{1}} m_{\Lambda_{1}}^{\prime}}^{\Lambda_{1}}(\sigma) \\
= & C_{A, m_{A}^{\prime \prime}, A, m_{A}^{\prime \prime \prime}}^{\tau_{\Lambda_{1}}, \Lambda_{1}, m_{m_{1}^{\prime \prime}}^{\prime \prime}} D_{m_{\Lambda_{1}}{m_{\Lambda}}_{1}}^{\Lambda_{1}}\left(\sigma^{-1}\right) D_{m_{\Lambda_{1}} m_{\Lambda_{1}}^{\prime}}^{\Lambda_{1}}(\sigma) \sum_{b} D_{A, m_{A}^{\prime \prime} i, m_{A}^{\prime \prime \prime} j}^{\gamma}\left(b^{*}\right) \\
= & C_{A, m_{A}^{\prime \prime}, A, m_{A}^{\prime \prime \prime}}^{\tau_{\Lambda_{1}}, \Lambda_{1}, m_{\Lambda_{1}}^{\prime}} \sum_{b} D_{A, m_{A}^{\prime \prime} i, m_{A}^{\prime \prime \prime} j}^{\gamma}\left(b^{*}\right) .
\end{aligned}
$$

The final step comes from the following equation

$$
\begin{aligned}
& C_{A, m_{A}, A, m_{A}^{\prime}}^{\tau_{\Lambda_{1}}, \Lambda_{1}, m_{\Lambda_{1}}} D_{m_{A} m_{A}^{\prime \prime}}^{A}(\sigma) D_{m_{A}^{\prime \prime \prime} m_{A}^{\prime}}^{A}\left(\sigma^{-1}\right) \\
= & C_{A, m_{A}, A, m_{1}^{\prime}}^{\tau_{\Lambda_{1}}, \Lambda_{1}, m_{\Lambda_{1}}} D_{m_{A}^{\prime \prime} m_{A}}^{A}\left(\sigma^{-1}\right) D_{m_{A}^{\prime \prime \prime} m_{A}^{\prime}}^{A}\left(\sigma^{-1}\right) \\
= & C_{A, m_{A}^{\prime \prime}, A, m_{A}^{\prime \prime \prime}}^{\tau_{\Lambda_{1}}, \Lambda_{1}, m_{\Lambda_{1}^{\prime \prime}}^{\prime \prime}} D_{m_{\Lambda_{1}}^{\prime \prime} m_{\Lambda_{1}}}^{\Lambda_{1}}\left(\sigma^{-1}\right) .
\end{aligned}
$$

See appendix A in [13] for the derivation.

\section{References}

[1] Y. Kimura and S. Ramgoolam, "Branes, Anti-Branes and Brauer Algebras in GaugeGravity duality," JHEP 0711 (2007) 078 [arXiv:0709.2158 [hep-th]].

[2] Y. Kimura and S. Ramgoolam, "Holomorphic maps and the complete 1/N expansion of 2D SU(N) Yang-Mills," JHEP 0806 (2008) 015 [arXiv:0802.3662 [hep-th]].

\footnotetext{
12 This comes from the expression of $b^{*}$ in (3.27) of [1]. with the assist of $\Sigma\left(\sigma b \sigma^{-1}\right)=\sigma \Sigma(b) \sigma^{-1}$, where $\Sigma(\sigma b \tau)=\sigma \Sigma(b) \tau$ is not true.
} 
[3] Y. Kimura and S. Ramgoolam, "Enhanced symmetries of gauge theory and resolving the spectrum of local operators," Phys. Rev. D 78 (2008) 126003 arXiv:0807.3696 [hep-th]].

[4] A. Hashimoto, S. Hirano and N. Itzhaki, "Large branes in AdS and their field theory dual," JHEP 0008 (2000) 051 arXiv:hep-th/0008016.

[5] S. Corley, A. Jevicki and S. Ramgoolam, "Exact correlators of giant gravitons from dual N = 4 SYM theory," Adv. Theor. Math. Phys. 5 (2002) 809 [arXiv:hep-th/0111222].

[6] D. Berenstein, "A toy model for the AdS/CFT correspondence," JHEP 0407 (2004) 018 arXiv:hep-th/0403110].

[7] H. Lin, O. Lunin and J. M. Maldacena, "Bubbling AdS space and 1/2 BPS geometries," JHEP 0410 (2004) 025 arXiv:hep-th/0409174.

[8] A. Ghodsi, A. E. Mosaffa, O. Saremi and M. M. Sheikh-Jabbari, "LLL vs. LLM: Half BPS sector of N = 4 SYM equals to quantum Hall system," Nucl. Phys. B 729 (2005) 467 arXiv:hep-th/0505129].

[9] Y. Takayama and A. Tsuchiya, "Complex matrix model and fermion phase space for bubbling AdS geometries," JHEP 0510 (2005) 004 arXiv:hep-th/0507070].

[10] J. McGreevy, L. Susskind and N. Toumbas, "Invasion of the giant gravitons from anti-de Sitter space," JHEP 0006 (2000) 008 arXiv:hep-th/0003075].

[11] M. T. Grisaru, R. C. Myers and O. Tafjord, "SUSY and Goliath," JHEP 0008 (2000) 040 [arXiv:hep-th/0008015].

[12] V. Balasubramanian, M. Berkooz, A. Naqvi and M. J. Strassler, "Giant gravitons in conformal field theory," JHEP 0204 (2002) 034 arXiv:hep-th/0107119.

[13] T. W. Brown, P. J. Heslop and S. Ramgoolam, "Diagonal multi-matrix correlators and BPS operators in N=4 SYM," JHEP 0802 (2008) 030 [arXiv:0711.0176 [hep-th]].

[14] R. Bhattacharyya, S. Collins and R. d. M. Koch, "Exact Multi-Matrix Correlators," JHEP 0803 (2008) 044 arXiv:0801.2061 [hep-th]].

[15] S. Ramgoolam, "Schur-Weyl duality as an instrument of Gauge-String duality," AIP Conf. Proc. 1031 (2008) 255 [arXiv:0804.2764 [hep-th]].

[16] R. Bhattacharyya, R. de Mello Koch and M. Stephanou, "Exact Multi-Restricted Schur Polynomial Correlators," JHEP 0806 (2008) 101 [arXiv:0805.3025 [hep-th]].

[17] T. W. Brown, P. J. Heslop and S. Ramgoolam, "Diagonal free field matrix correlators, global symmetries and giant gravitons," JHEP 0904 (2009) 089 arXiv:0806.1911] [hepth]]. 
[18] S. Corley and S. Ramgoolam, "Finite factorization equations and sum rules for BPS correlators in N = 4 SYM theory," Nucl. Phys. B 641 (2002) 131 arXiv:hep-th/0205221.

[19] T. S. Tai, "Classical c=1 Tachyon Scattering and 1/2-BPS Correlators," Prog. Theor. Phys. 117 (2007) 795 arXiv:hep-th/0701086].

[20] R. de Mello Koch and R. Gwyn, "Giant graviton correlators from dual SU(N) super Yang-Mills theory," JHEP 0411 (2004) 081 arXiv:hep-th/0410236|.

[21] T. W. Brown, "Half-BPS SU(N) correlators in N = 4 SYM," JHEP 0807 (2008) 044 arXiv:hep-th/0703202.

[22] D. J. Gross and W. Taylor, "Two-dimensional QCD is a string theory," Nucl. Phys. B 400 (1993) 181 arXiv:hep-th/9301068]; "Twists and Wilson loops in the string theory of two-dimensional QCD," Nucl. Phys. B 403 (1993) 395 arXiv:hep-th/9303046].

[23] M. Hamermesh, "Group theory and it application to physical problems," AddisonWesley Publishing.

[24] A. P. Polychronakos, "Physics and mathematics of Calogero particles," J. Phys. A 39 (2006) 12793 arXiv:hep-th/0607033.

[25] V. Balasubramanian, B. Czech, K. Larjo and J. Simon, "Integrability vs. information loss: A simple example," JHEP 0611 (2006) 001 arXiv:hep-th/0602263].

[26] V. Balasubramanian, B. Czech, K. Larjo, D. Marolf and J. Simon, "Quantum geometry and gravitational entropy," JHEP 0712 (2007) 067 [arXiv:0705.4431 [hep-th]].

[27] Y. Kimura, S. Ramgoolam and D. Turton, "Free particles from Brauer algebras in complex matrix models," arXiv:0911.4408 [hep-th].

[28] J. M. Maldacena and A. Strominger, "AdS(3) black holes and a stringy exclusion principle," JHEP 9812 (1998) 005 arXiv:hep-th/9804085.

[29] A. Jevicki and S. Ramgoolam, "Non-commutative gravity from the AdS/CFT correspondence," JHEP 9904 (1999) 032 [arXiv:hep-th/9902059].

[30] V. Balasubramanian, D. Berenstein, B. Feng and M. x. Huang, "D-branes in Yang-Mills theory and emergent gauge symmetry," JHEP 0503 (2005) 006 arXiv:hep-th/0411205.

[31] R. de Mello Koch, J. Smolic and M. Smolic, "Giant Gravitons - with Strings Attached (I)," JHEP 0706 (2007) 074 arXiv:hep-th/0701066].

[32] R. de Mello Koch, J. Smolic and M. Smolic, "Giant Gravitons - with Strings Attached (II)," JHEP 0709 (2007) 049 arXiv:hep-th/0701067. 
[33] D. Bekker, R. de Mello Koch and M. Stephanou, "Giant Gravitons - with Strings Attached (III)," JHEP 0802 (2008) 029 [arXiv:0710.5372 [hep-th]].

[34] M. Bianchi, B. Eden, G. Rossi and Y. S. Stanev, "On operator mixing in N = 4 SYM," Nucl. Phys. B 646 (2002) 69 arXiv:hep-th/0205321.

[35] G. Arutyunov, S. Penati, A. C. Petkou, A. Santambrogio and E. Sokatchev, "Nonprotected operators in N = 4 SYM and multiparticle states of AdS(5) SUGRA," Nucl. Phys. B 643 (2002) 49 arXiv:hep-th/0206020.

[36] N. Beisert, C. Kristjansen, J. Plefka, G. W. Semenoff and M. Staudacher, "BMN correlators and operator mixing in $\mathrm{N}=4$ super Yang-Mills theory," Nucl. Phys. B 650 (2003) 125 arXiv:hep-th/0208178.

[37] N. R. Constable, D. Z. Freedman, M. Headrick and S. Minwalla, "Operator mixing and the BMN correspondence," JHEP 0210 (2002) 068 arXiv:hep-th/0209002.

[38] M. Bianchi, G. Rossi and Y. S. Stanev, "Surprises from the resolution of operator mixing in N = 4 SYM," Nucl. Phys. B 685 (2004) 65 arXiv:hep-th/0312228.

[39] S. Bellucci, P. Y. Casteill, J. F. Morales and C. Sochichiu, "Spin bit models from nonplanar N = 4 SYM," Nucl. Phys. B 699 (2004) 151 arXiv:hep-th/0404066]; S. Bellucci, P. Y. Casteill, A. Marrani and C. Sochichiu, "Spin bits at two loops," Phys. Lett. B 607 (2005) 180 arXiv:hep-th/0411261.

[40] T. W. Brown, "Permutations and the Loop," JHEP 0806 (2008) 008 [arXiv:0801.2094 [hep-th]].

[41] N. Beisert, "The complete one-loop dilatation operator of $\mathrm{N}=4$ super Yang-Mills theory," Nucl. Phys. B 676, 3 (2004) arXiv:hep-th/0307015.

[42] N. Beisert, "The dilatation operator of $\mathrm{N}=4$ super Yang-Mills theory and integrability," Phys. Rept. 405, 1 (2005) arXiv:hep-th/0407277].

[43] A. Ram, "Representation theory," Thesis, Univ. Cal. San Diego 1991. 